\title{
In vivo gene editing of outer hair cells prevents progressive hearing loss in a dominant-negative KCNQ4 murine model
}

\section{Byunghwa Noh}

Yonsei University College of Medicine

John Hoon Rim

Yonsei University

Ramu Gopalappa

Yonsei University College of Medicine

Haiyue Lin

Yonsei University College of Medicine

Kyu Min Kim

Yonsei University College of Medicine

Heon Yung Gee

Yonsei University College of Medicine https://orcid.org/0000-0002-8741-6177

Jae Young Choi

Yonsei University College of Medicine

Hyongbum Henry Kim

Yonsei University College of Medicine https://orcid.org/0000-0002-4693-738X

Jinsei Jung ( $\sim$ JSJUNG@yuhs.ac)

Yonsei University College of Medicine https://orcid.org/0000-0003-1906-6969

\section{Article}

Keywords: Adult-onset hearing loss, outer hair cell, DFNA2, KCNQ4, Cas9s, gene editing

Posted Date: February 4th, 2021

DOl: https://doi.org/10.21203/rs.3.rs-152704/v1

License: (c) (i) This work is licensed under a Creative Commons Attribution 4.0 International License.

Read Full License 


\title{
In vivo gene editing of outer hair cells prevents progressive hearing
}

\section{loss in a dominant-negative $K C N Q 4$ murine model}

Byung Wha Noh ${ }^{1,2,7}$, John Hoon Rim ${ }^{2,3,7}$, Ramu Gopalappa ${ }^{3,4,7}$, Haiyue Lin ${ }^{1,2}$, Kyu Min Kim ${ }^{1,2}$, Heon Yung Gee ${ }^{2,4 *}$, Jae Young Choi ${ }^{1,2,3, *}$, Hyongbum Henry Kim ${ }^{2,3,4,5 *}$, Jinsei Jung ${ }^{1,2 *}$

\author{
Affiliations: \\ ${ }^{1}$ Department of Otorhinolaryngology, Graduate School of Medical Science, Brain Korea 21 \\ Project, Yonsei University College of Medicine, Seoul, Republic of Korea \\ ${ }^{2}$ Institute for Yonsei Ear Science, Seoul, Republic of Korea \\ ${ }^{3}$ Severance Biomedical Science Institute, Yonsei University College of Medicine, Seoul, \\ Republic of Korea \\ ${ }^{4}$ Department of Pharmacology, Graduate School of Medical Science, Brain Korea 21 Project, \\ Yonsei University College of Medicine, Seoul, Republic of Korea \\ ${ }^{5}$ Center for Nanomedicine, Institute for Basic Science, Seoul, Republic of Korea \\ ${ }^{6}$ Yonsei-IBS Institute, Yonsei University, Seoul, Republic of Korea \\ ${ }^{7}$ These authors contributed equally.
}

*Correspondence: jsjung@yuhs.ac (JJ); hkim1@yuhs.ac (HHK); jychoi@yuhs.ac (JYC); hygee@yuhs.ac (HYG) 


\begin{abstract}
Adult-onset hearing loss (AHL)—including presbycusis-caused by outer hair cell (OHC) degeneration is the most common sensorial disorder. Despite the high prevalence of AHL and wide therapeutic window, no targeted therapy is currently available. Here, we generated a mouse model harboring $K c n q 4^{\mathrm{W} 276 \mathrm{~S} /+}$ to recapitulate DFNA2, a common genetic form of progressive hearing loss caused by degenerating OHCs. By comprehensively optimizing guide RNAs, Cas9s, vehicles, and delivery routes, we found that in vivo gene editing using dual adeno-associated virus packaging in OHCs via the round window membrane significantly improved auditory function. We developed a new technique using live-cell imaging to measure the membrane potential of the OHCs and demonstrated that our approach resulted in more hyperpolarized, steady-state OHCs, indicative of elevated KCNQ4 channel activity. These findings can help develop targeted therapy for AHL and support the use of CRISPR-based gene therapy to rectify defects in OHCs.
\end{abstract}

Keywords: Adult-onset hearing loss, outer hair cell, DFNA2, KCNQ4, Cas9s, gene editing 


\section{Introduction}

Hearing loss is a common sensory disorder ${ }^{1-3}$ that affects 466 million people or approximately $6.1 \%$ of the world population (World Health Organization, 2018; https://www.who.int/pbd/deafness/estimates). The risk of hearing impairment increases with age, and the number of individuals with adult-onset hearing loss (AHL) is growing each decade; for instance, approximately $60 \%$ of individuals aged $70-79$ years suffer from hearing loss for sounds $\geq 25 \mathrm{~dB}^{3}$. In $2020,56 \%$ of individuals $>70$ years of age are expected to experience AHL; moreover, this proportion is expected to increase to $67 \%$ by $2060^{4}$. However, no treatment is currently available for AHL.

AHL can develop due to many risk factors (genetic and environmental) 5 . Lifetime acoustic noise exposure is often the primary cause of age-related hearing loss ${ }^{6}$. Moreover, alterations in genes that encode potassium channels located in the cochlea-the main component associated with the development of AHL—can influence individual susceptibility to noise exposure ${ }^{7,8}$. The leading cause of AHL is the degeneration of outer hair cells (OHCs), often triggered by genetic vulnerability to noise exposure ${ }^{6,9}$. In this respect, functionally restoring OHCs is indispensable for preventing and treating AHL.

Genome editing technologies, including Cas9 nucleases, base editors, and prime editors, are emerging as tools for treating genetic deficits responsible for causing various diseases ${ }^{10,11}$. In particular, Cas9 nucleases are effective and useful for disrupting dominant-negative alleles, whereas base and prime editors are potentially useful for rescuing recessive loss-of-function mutations. While gene replacement or silencing therapies for hearing loss have been attempted in several mouse models ${ }^{12-19}$, in vivo gene editing faces numerous therapeutic hurdles, including the selection of suitable vehicles and delivery routes to efficiently and safely deliver the gene editing 
system $^{20,21}$. Previously, in vivo gene editing with Cas9 nuclease or base editor had been employed in mice with transmembrane channel-like 1 gene $(T m c 1)$ variants to restore hearing ${ }^{22-}$

24. However, previous in vivo gene editing techniques have not provided sufficient transduction rates in OHCs; therefore, in vivo gene editing systems need to be further optimized before their application in a clinical setting ${ }^{25}$.

This study attempted to employ in vivo gene editing to treat AHL resulting from $\mathrm{OHC}$ degeneration using a newly established murine model with a dominant-negative effect of a missense mutation (p.W276S) in the voltage-gated potassium channel subfamily Q member 4 gene (Kcnq4). Moreover, we comprehensively optimized the efficiency, safety, and delivery route of the Cas 9 nuclease system to improve the feasibility of using in vivo genome editing to treat AHL.

\section{Results}

\section{Selection of KCNQ4 for in vivo gene editing}

We first attempted to identify genetic predispositions that contribute to the development of AHL. To this end, we enrolled 213 patients with AHL and performed whole-exome or targeted deafness panel sequencing. The results showed that genetic mutations accounted for $33.8 \%$ of AHL, and KCNQ4 was the most frequently mutated gene in this cohort (Fig. 1a and Supplementary Table 1). Indeed, DFNA2 (deafness, autosomal dominant type 2) associated with KCNQ4 mutations is one of the most common causes of autosomal dominant hearing loss ${ }^{26,27}$. We selected $K C N Q 4$ as a target for in vivo gene editing based the following reasons: 1 ) KCNQ4 is also associated with age-related hearing loss and noise-induced hearing loss ${ }^{8,28} ; 2$ ) DFNA2 usually exhibits progressive hearing loss, which provides a broad therapeutic window; 
and 3) though KCNQ4 is exclusively expressed at the basolateral surface of OHCs (Fig. 1b), it is indispensible for maintaining the electrolyte milieu of the endolymph via potassium recycling in the cochlea ${ }^{29}$.

Among over 40 DFNA2-linked $K C N Q 4$ mutations, we chose c.830G $>\mathrm{C}$ (p.W276S) as a candidate for in vivo gene editing because this mutation is commonly found worldwide ${ }^{30-32}$. Subsequently, knock-in mice harboring the Kcnq4 c.830G $>\mathrm{C}$ allele and a silent mutation at nucleotide position 810 were generated (Fig. 1c). The silent mutation (c.810C >A; p.S269=) not only facilitated genotyping by making only the mutant allele susceptible to Nde1 but also discriminated between the edited mutant alleles containing the indel and the wild-type alleles affected by Cas9-single guide-RNA (sgRNA) in in vivo gene editing experiments.

We found that Kcnq $4^{\mathrm{W} 276 \mathrm{~S} / \mathrm{W} 276 \mathrm{~S}}$ (homozygote) and Kcnq $4^{\mathrm{W} 276 \mathrm{~S} /+}$ (heterozygote) mice distintly exhibited moderate hearing loss in the auditory brainstem response (ABR) at P21 (postnatal 21 d) (Fig. 1d). A progressive hearing loss phenotype was also validated in the followup hearing function tests performed at P49 and P77. Hearing loss was attributed to the degeneration of OHCs, particularly in the high-frequency region in the cochlea (Fig. 1e and f). The morphology of stereocilia in the surviving hair cells of Kcnq $4^{\mathrm{W} 276 \mathrm{~S} /+}$ mice was normal (similar to that in wild-type mice; Supplementary Fig. 1). Hearing thresholds at all frequencies reached almost scale-out levels at $\mathrm{P} 49$, which was determined as the time point of deep sequencing in the subsequent experiments (Fig. 1d). 
Fig. 1

Adult-onset hearing loss $(n=213)$

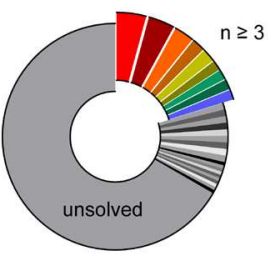

b

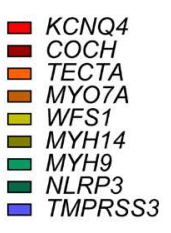

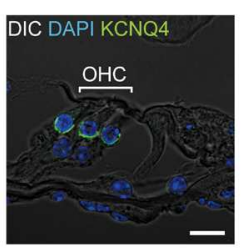

Kcnq4

GRCm39/mm39; chr4:120572970 C>G

NM_001081142 c.830G >C (exon5)

$$
\begin{array}{ll}
\text { c. } 810 \mathrm{C}>\mathrm{A} ; & \text { c. } 830 \mathrm{G}>\mathrm{C} ; \\
\text { p.S269 } & \text { p.W276S }
\end{array}
$$

GACTTCTCCTCATATGCCGACTCGCTCTGGTCGGGGACGGTG

Ndel re-cut

prevention

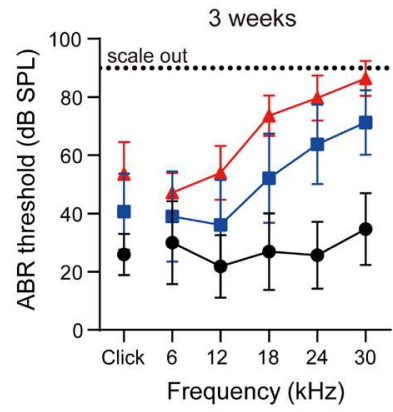

e

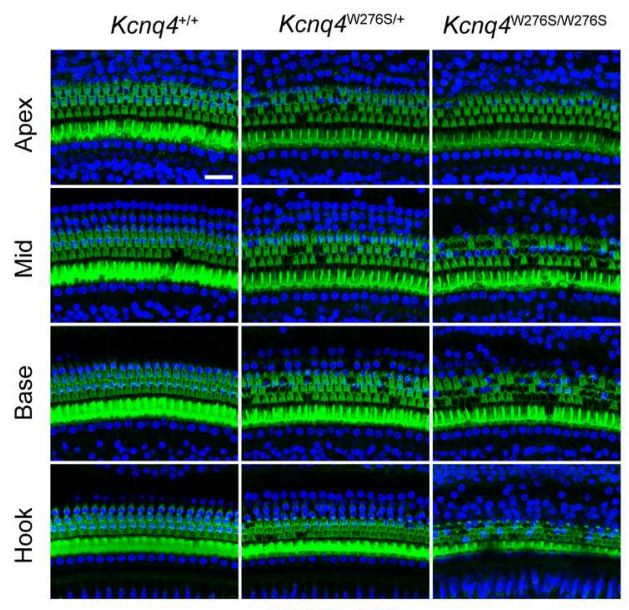

DAPI Phalloidin
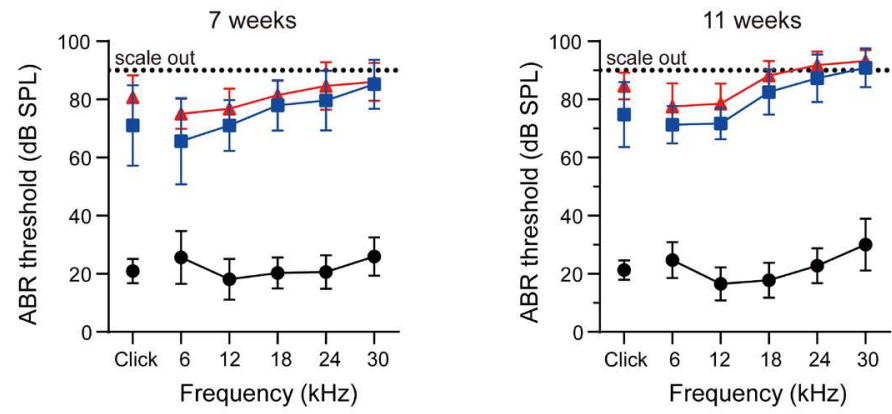

f

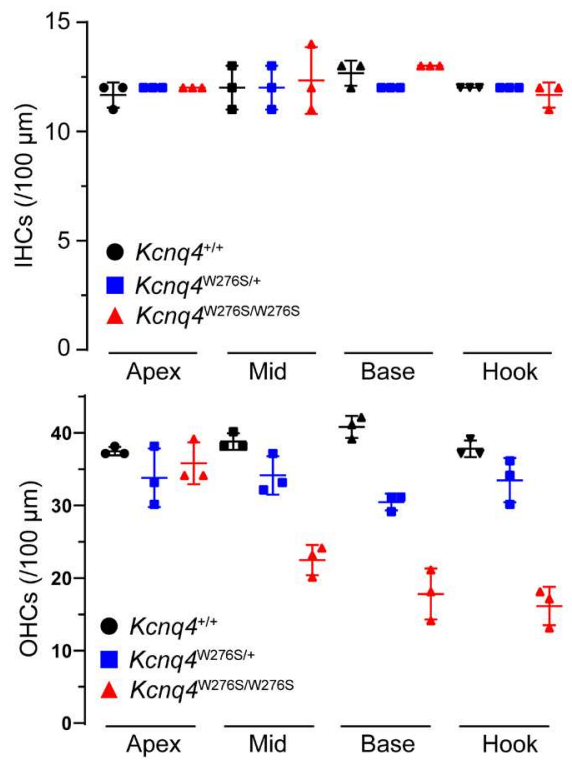

Fig. 1. Mutant KCNQ4 as a common cause of adult-onset hearing loss (AHL) in humans and mice. (a) In 213 patients with AHL, whole or targeted exome sequencing was performed. Of the 213 individuals, 33.8\% (72 patients) had a genetic diagnosis of AHL. Among them, KCNQ4 $(n=9)$ was most commonly mutated gene. (b) KCNQ4 expression in the basolateral surface of the outer hair cells (OHCs) in the organ of Corti of the inner ear of a 3-week-old wild-type mouse. Scale bar, $10 \mu \mathrm{m}$. (c) Sequence data for the Kcnq4 mutant mouse model used. A pathogenic missense mutation in Kcnq4 (c.830G $>$ C) was targeted for gene editing. Prior to target mutation, an additional synonymous variant (c.810C $>$ A) was introduced at nucleotide position 20 because this synonymous variant was utilized for the identification of the original mutant allele after Cas9 editing at the target mutation site; moreover, this variant prevented the Ndel enzyme from cutting within this position, enabling the use of Nde1 restriction enzymes for genotyping heterozygote mutant mice. (d) Characteristics associated with auditory function in Kcnq4 heterozygote mutant mice. (e) Cross-sectional images of the cochlea at the apex, mid, base, and hook regions from 3-week-old wild-type, heterozygote mutant, and homozygote mutant mice. Immunostaining was performed with DAPI (blue) and phalloidin (green). Scale bar, $20 \mu \mathrm{m}$. (f) Number of live inner hair cells (IHCs) and outer hair cells (OHCs) in sections indicated in (d). 


\section{Optimizing the targeting efficiency of the Cas9-sgRNA system for Kcnq4 c.830G>C}

To select active and efficient sgRNA for target Kcnq4 mutation (c.830G $>$ C) editing, all possible sgRNA target sequences with a protospacer-adjacent motif (PAM; 5'-NGG-3') downstream of the mutation locus were designed (Fig. 2a and Supplementary Fig. 2a). The gene editing scores for each sgRNA were determined using the deepSpCas 9 prediction program ${ }^{33}$, which confirmed that the combination of SpCas9 (Strepotococcus pyogenes) wild type and T3 was the most efficient among the various types of SpCas9 (Supplementary Table 2). To elucidate the activity of these sgRNAs, we generated a reporter cell line expressing the Kcnq4 mutation (c.830G>C) target sequence with a dual-fluorescent reporter construct. The presence of the reporter was detected by a sole RFP signal, whereas nuclease activity was analyzed by simultaneous GFP and RFP fluorescence (Fig. 2b and Supplementary Fig. 2b). Next, we isolated the genomic DNA from reporter cells in the transfected pooled cells and performed T7E1 assay and NGS analysis to evaluate the indel frequency at target sites. The mutation frequencies at the target sites ranged from $31-34 \%$ and $20-24 \%$ as per the T7E1 assay and NGS, respectively (Fig. 2c).

To determine the wild type off-target cleavage activity of sgRNA, genomic DNA from wild-type Neuro2A cells transfected with SpCas9 and individual sgRNA expressing plasmids were analyzed using the T7E1 assay. We observed indel frequencies of $9 \%, 41 \%, 0 \%$, and $47 \%$ for each respective sgRNA (T1-T4) in the wild type target sites (Fig. 2d). Finally, sgRNA-T3 was selected for subsequent in vivo gene editing because it showed no cleavage activity for the wild-type target locus while exhibiting high-cleavage activity for the c.830G $>$ C mutation target sequence (Fig. 2c, d).

Next, we optimized both adeno-associated virus (AAV) and ribonucleotide complex (RNP) materials for in vivo intracochlear delivery. For AAV packaging, we chose the 
AAV2/Anc80L65 serotype due to its high efficiency in the $\mathrm{OHCs}^{34,35}$. We designed split Cas9 and gRNA-T3 into two plasmids (Supplementary Fig. 3a-d). The final injected AAV titer was estimated to be $1.00 \times 10^{9}$ genomic copies in one cochlea with a volume of $1 \mu \mathrm{L}$ (Supplementary Fig. 3e). For RNP packaging, we optimized the liposome packaging protocol using Lipofectamine 2000 as it has been reported to have high efficacy for RNP application in the cochlea (Supplementary Fig. 4a) ${ }^{22}$. After analyzing various titer combinations of the SpCas9 protein, sgRNA, and Lipofectamine 2000, we determined the optimal RNP mixture ratio and obtained up to $32.2 \%$ indel efficiency under in vitro conditions (Supplementary Fig. 4b). Finally, we used $1.5 \mu \mathrm{g}$ SpCas9 and $1.0 \mu \mathrm{g}$ sgRNA-T3 for RNP packaging in each mouse.

To determine the best delivery route, we investigated the in vivo efficiency of viral injection through the round window $(\mathrm{RW})$, scala media $(\mathrm{SM})$, posterior semi-circular canal (PSCC), and utricle using AAV2/Anc80L65-eGFP (Supplementary Fig. 5a) ${ }^{36}$. The SM route via cochleostomy or RW route showed the highest eGFP expression levels in the OHCs across all frequency regions (Supplementary Fig. 5b). Therefore, we mainly injected RNP into the SM via cochleostomy or AAV into the RW. Although cochleostomy damaged the hearing function in some cases due to technical difficulties, in most cases hearing loss was not induced in wild-type mice when cochleostomy was appropriately performed (Supplementary Fig. 6). 
Fig. 2

a

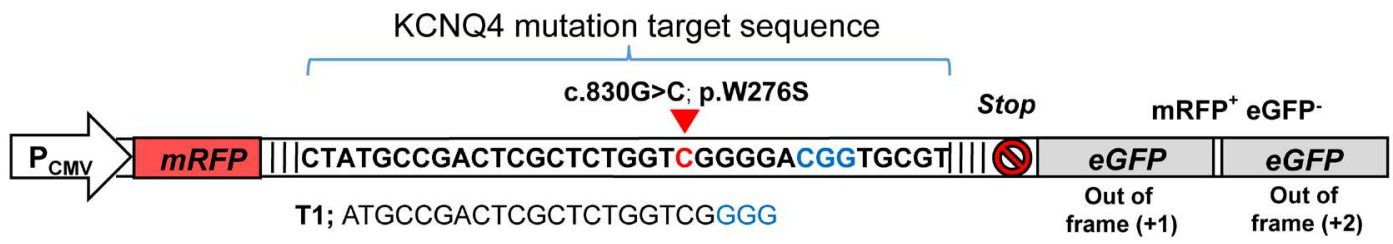

T2; TATGCCGACTCGCTCTGGTCGGG

T3; CGACTCGCTCTGGTCGGGGACGG

T4; CTATGCCGACTCGCTCTGGTCGG

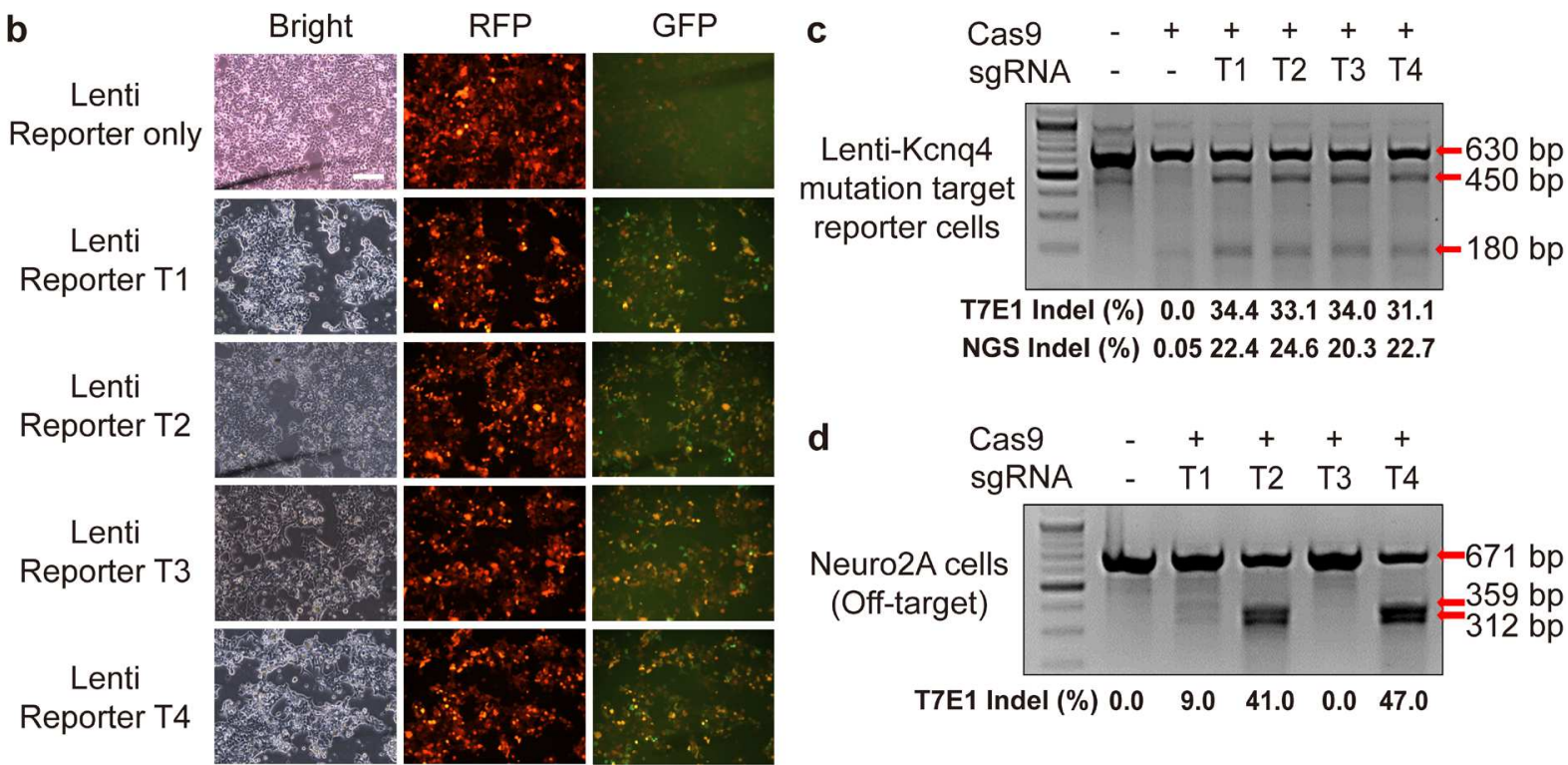

Fig. 2. Optimization of sgRNA for in vivo gene editing. (a) Schematic representation of the reporter construct for sgRNA selection. Protospacer-adjacent motif (PAM) sequences of each sgRNA are in blue. (b) Fluorescent microscopy images of the reporter cell lines after transfection of the plasmid encoding Cas9 and each sgRNA targeting the Kcnq4 mutation target region in the reporter cells. Scale bar, $50 \mu \mathrm{m}$. (c) Target editing efficiency of sgRNA candidates determined by the T7E1 assay and deep sequencing analysis. The top band is uncut DNA at $630 \mathrm{bp}$; the two smaller cleaved bands are edited DNA (450 bp and $180 \mathrm{bp}$ ). (d) Off-target activity screening for sgRNA candidates. 


\section{Phenotypic correction of Kcnq4 p.W276S mice by CRISPR/Cas9 gene editing}

After injecting the appropriate AAV and RNP packages into Kcnq4W276S/+ mice within P4 d, phenotypic rescue was evaluated by ABR and distortion-product otoacoustic emission (DPOAE) at 3 and 7 weeks after injection (Fig. 3a). The representative traces of ABR waves 7 weeks after injection are shown in response to varying sound intensity of $6-\mathrm{kHz}$ tone bursts (Fig. 3b). In the AAV-injected ears, the ABR threshold, P1 amplitude, and P1 latency were significantly improved at all frequencies compared with those of uninjected ears (Fig. 3c and Supplementary Fig. 7a-c). The amplitude in DPOAE in the AAV-injected ears was significantly increased at frequencies corresponding to the 12- and 16-kHz regions (Fig. 3d). Meanwhile, in the RNPinjected mice, improvements in ABR threshold, P1 amplitude, and P1 latency were comparable to or less than those in the AAV-injected mice (Fig. 3e and Supplementary Fig. 7d-f). However, the amplitudes obtained by DPOAE in the RNP-vehicle-injected ears were not significantly improved, except in the 12-kHz region (Fig. 3f). Taken together, both AAV- and RNP-vehicle injection effectively restored hearing function in $K c n q 4^{\mathrm{W} 276 \mathrm{~S} /+}$ mice. 
Fig. 3

a

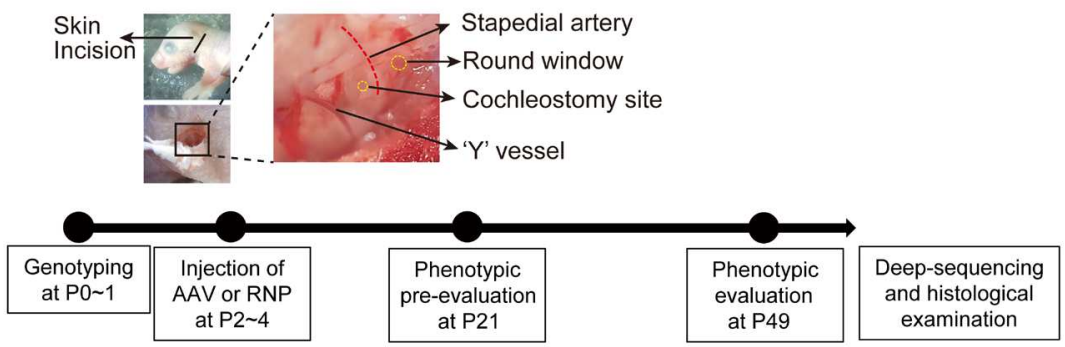

b
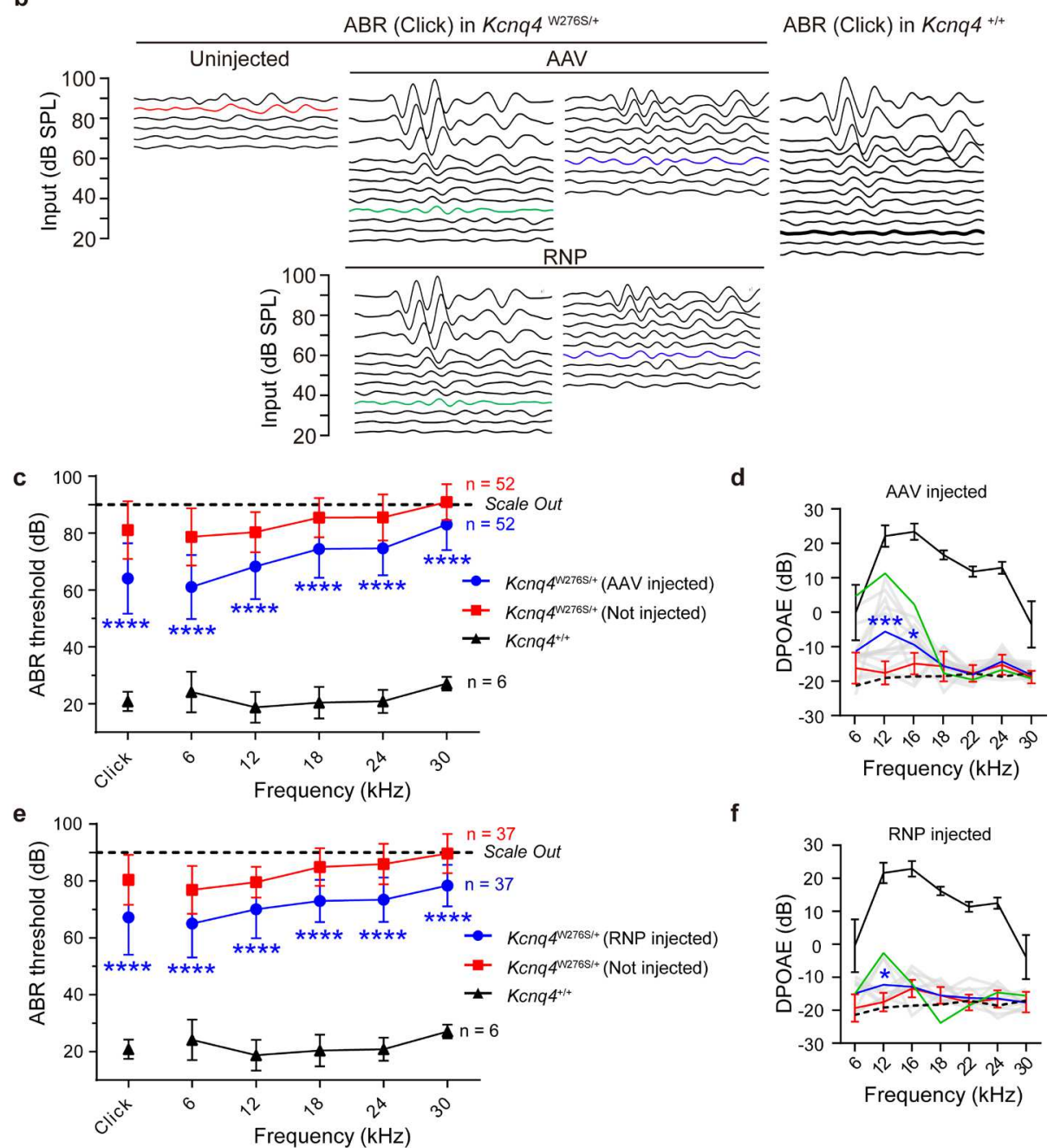

$f$

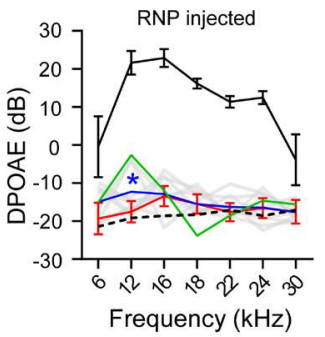

Fig. 3. Phenotypic rescue of hearing loss by AAV and RNP injections in $K c n q 4^{+/ W 276 S}$ mice. (a) Experimental workflow of injection procedures to examine the phenotype and determine gene editing efficacy. (b) Auditory brainstem response (ABR) waveform patterns of contralateral uninjected, AAV-injected, and RNP-injected cochlea from 7-week-old Kcnq4 mutant and wild-type mice (control). The best (green) and median (blue) recovery degrees are shown. (c) Comparative analysis of ABR thresholds across all frequencies in AAV-injected and uninjected cochlea of mutant and wild-type control mice. (d) Distortionproduct otoacoustic emission (DPOAE) thresholds in AAV-injected mutant, uninjected mutant, and wild-type mice. The best (green) and mean (blue) corrected cochleae are presented, along with the other AAV-injected cochleae (gray). (e) Comparative analysis of ABR thresholds across all frequencies in RNP-injected mutant, uninjected mutant, and wild type cochleae. (f) DPOAE thresholds of RNP-injected mutant, uninjected mutant, and wild-type mice. The best (green) and mean (blue) corrected cochleae are presented with other RNP-injected cochleae (gray). ${ }^{*} p<0.05 ; * * * p<0.001 ; * * * *<0.0001$. 


\section{Gene editing efficiency in phenotypically corrected mice}

Gene editing efficiency was evaluated using allele-specific indel analysis of phenotypically corrected mice. When the best edited in vivo sample was compared with an ex vivo sample, only $0.6 \%$ mutant alleles were corrected in the AAV-injected case, showing diverse indel patterns (similar to ex vivo samples, 1.5\%; Fig. 4a). Nevertheless, reproducible and relevant sequenced fragments having out-of-frame insertion and valid deletion patterns at the target cleavage site confirmed the validity of genotype correction. Furthermore, the fold changes in the editing rates correlated with the threshold changes in ABR after AAV and RNP injection (Fig. 4b). When gene editing efficiency rates were matched with the degree of hearing improvement (categorized as marked, moderate, mild, and minimal groups) based on the average differences in ABR thresholds between the injected and contralateral uninjected cochlea, significant correlation was observed in marked, moderate, and mild groups in the AAV-injected cases (Fig. 4c). In the RNPinjected cases, only the marked and moderate groups exhibited significant differences. Overall, the gene editing efficacy of the AAV-vehicle for rescuing hearing loss was more favorable and higher than that of the RNP-vehicle. Therefore, we investigated the mechanism responsible for this distinct phenotypic rescue in the AAV-injected mice.

\section{Effect of CRISPR/Cas9-induced ablation of mutant alleles on cochlear function}

We found that the viability of OHCs in the Kcnq $4^{\mathrm{W} 276 \mathrm{~S} /+}$ mice with improved hearing thresholds after AAV injection was not significantly different from that of OHCs in uninjected mice (Fig. 4d, e and Supplementary Fig. 8a). Similarly, there were no differences in KCNQ4 expression in the OHCs (Supplementary Fig. 8b), the extent of neurofilament innervated into hair cells (Supplementary Fig. 8c, d), and the number of spiral ganglion neurons (Supplementary Fig. 
9a, b) between the AVV-injected and uninjected groups 7 weeks after injection. Finally, no morphological difference was observed in the stereocilia of the IHCs and OHCs between the two groups (Supplementary Fig. 9c). Therefore, hearing restoration by the CRISPR/Cas9 system could not be explained by the survival of hair cells and neurons. 


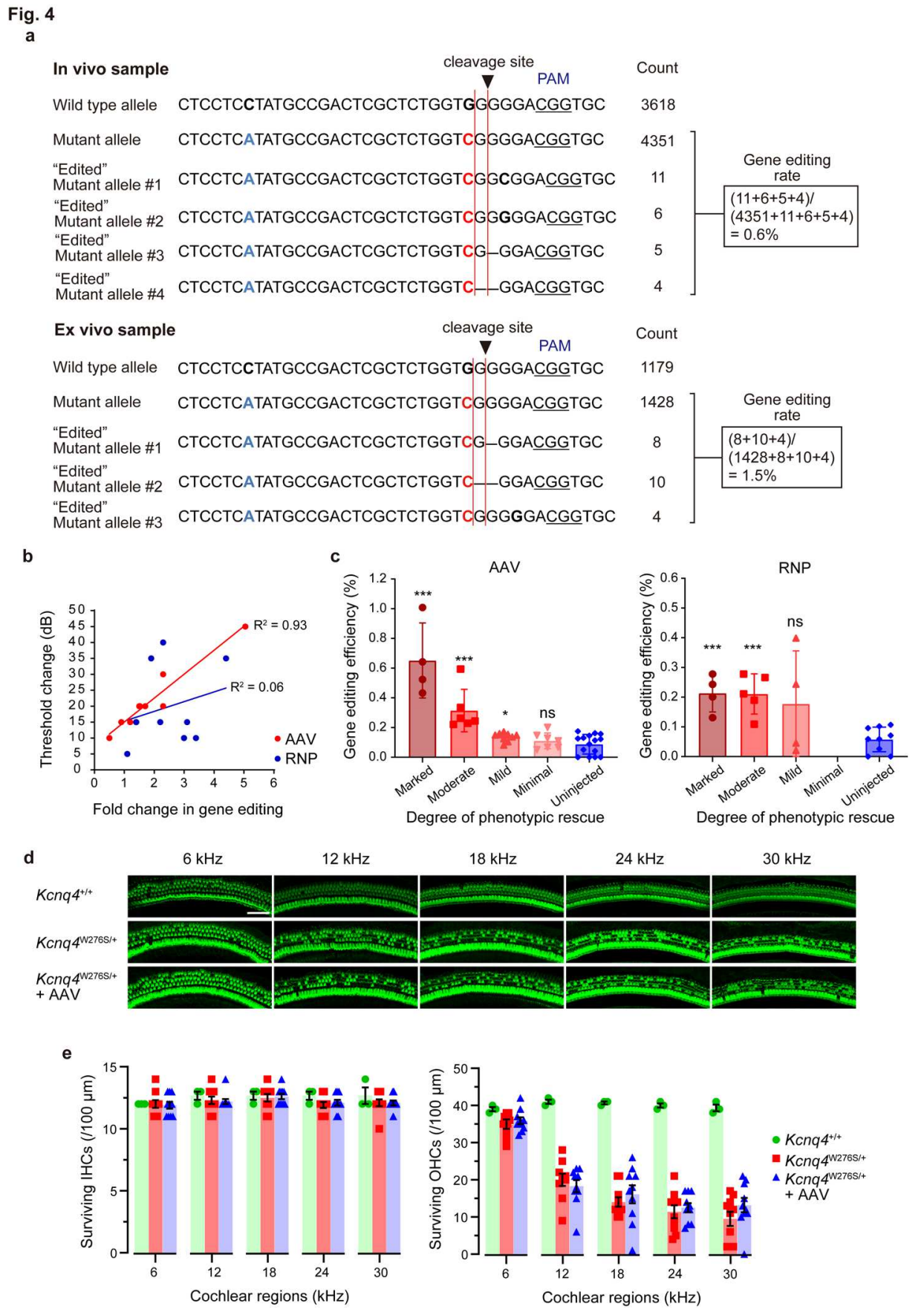

Fig. 4. In vivo gene editing by $\mathrm{AAV}$ and RNP injection and the corresponding degree of phenotypic rescue in $K c n q 4^{+/ W 276 S}$ mice. (a) Allele-specific gene editing rates obtained by deep sequencing. (b) Correlation between gene editing efficiency in terms of the fold change of correction rates and phenotypic rescue degrees evaluated by ABR threshold shift levels at click sound. Fold changes in mutant allele-specific correction rates in injected and contralateral cochleae of the same mouse showed a higher correlation trend $\left(\mathrm{R}^{2}=0.93\right)$ with ABR threshold shift in AAV than in RNP $\left(\mathrm{R}^{2}=0.06\right)$. (c) Gene editing efficiency according to phenotypic rescue degrees evaluated by ABR threshold shift levels across all frequencies. Phenotypic rescue degrees were classified into four subgroups based on average $\mathrm{dB}$ differences between injected and contralateral cochleae at five frequencies $(6$, $12,18,24$, and $30 \mathrm{kHz}$ ): marked: $\geq 15 \mathrm{~dB}$; moderate: $10-14 \mathrm{~dB}$; mild: 5-9 dB; and minimal: $\leq 5 \mathrm{~dB}) .{ }^{*} p<0.05 ; * * * p<0.001$; ns, not significant. (d) Hair cell survival patterns in the cochleae of wild-type, uninjected mutant, and injected mutant across five frequencies after immunostaining with phalloidin (green). Scale bar, $50 \mu \mathrm{m}$. (e) Number of live inner and outer hair cells in the cochleae of wild-type, uninjected mutant, and injected mutant mice across five frequencies $(p>0.05)$. 
Thus, we hypothesized that the surviving cells functionally differ with respect to mutant allele ablation. Therefore, we developed a novel ex vivo hair cell thallium imaging technique to reflect the state of membrane potential. Because the potassium (or thallium) influx into the hair cells via nonselective cationic channels is affected by the electrochemical gradient, the ion influx amount depends on the membrane potential. Therefore, more hyperpolarized cells are associated with faster thallium influx (Supplementary Fig. 10). Given that KCNQ4 in the basolateral surface can hyperpolarize hair cells ${ }^{29}$, we speculated that the restored activity of KCNQ4 by gene editing increases the influx of thallium ions through apical cationic channels. To evaluate the electrophysiological rescue of KCNQ4 by AAV injection, the uncapped cochlea incubated with FluxOR dye was visualized using fluorescence microscopy (Fig. 5a). We successfully observed that the thallium influx was exclusively dominant in the OHCs of $\mathrm{Kcnq}^{+/+}$mice (Fig. $5 \mathbf{b}$ and Supplementary Movie 1). In addition, the influx of the thallium ions depended on the leaky activity of mechano-electrical transduction (MET) channels at the apex during the resting steadystate (Fig. 5c). Because we did not induce mechanical stimulation to activate the hair cells, thallium influx solely depended on the membrane potential of the hair cells, regardless of the activation state of MET (i.e., TMC1) or KCNQ4 channels $^{37-39}$.

FluxOR signals increased more rapidly in the OHCs of mice with AAV injection than in those with mock injection (Fig. 5d). Both the OHCs and IHCs showed a significantly increased thallium influx slope after AAV injection (Fig. 5e, f). These findings indicate that the surviving hair cells in the Kcnq4 mutant mice are more hyperpolarized, similar to those in the wild-type mice after the mutant allele ablation by gene editing. 
Fig. 5

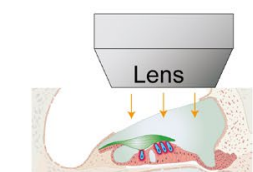

Organ of Corti

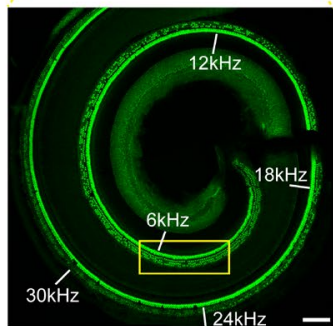

d

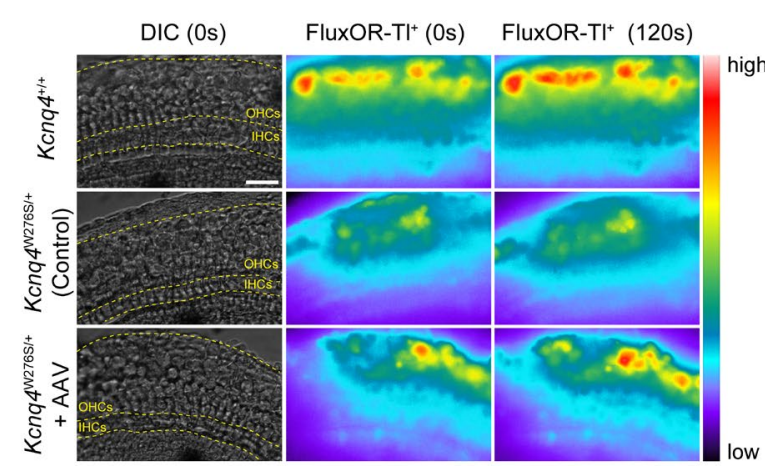

b

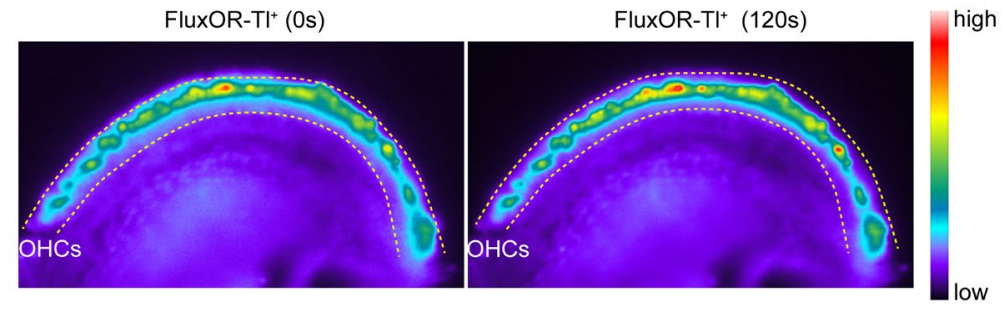

c $2.107-$ WT OHCS

1.95 - - WT OHCs + 10mM Quinine

$1.95-$ - WT OHCs + 10mM Quinine

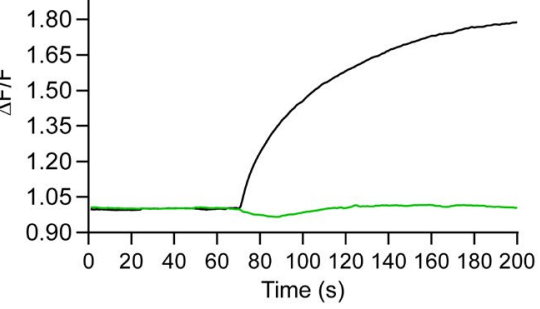

e

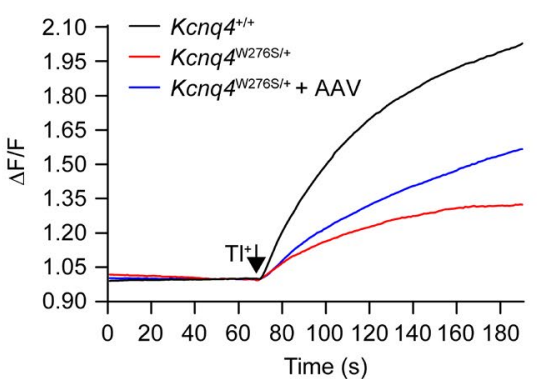

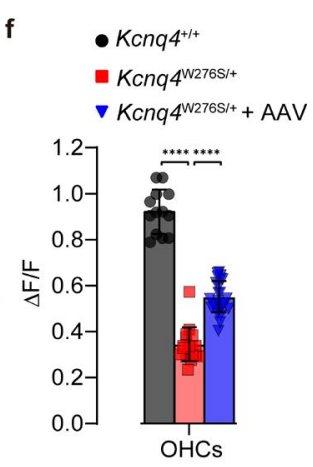

Fig. 5. Mechanisms responsible for phenotypic rescue in $K c n q 4^{+/ W 276 S}$ mice in response to gene editing. (a) Cochlea stained with phalloidin (green) of AAV-injected mutant mice euthanized at 7 weeks. Tonotopical regions are marked with the corresponding frequencies (white); $\mathrm{Tl}^{+}$flux assay was performed at the $6 \mathrm{kHz}$ region under a microscope. Scale bar, $100 \mu \mathrm{m}$. (b) Microscopy images of the organ of Corti (apex) of 7-week-old wild type mouse before the addition of $\mathrm{Tl}^{+}$and after 120 seconds. (c) Changes in fluorescence intensity values in the 6-kHz region of the cochlea of 7 -week-old wild-type mice after the addition of the stimulus buffer, including $\mathrm{Tl}^{+}$, during the thallium flux assay. In the negative control, $10 \mathrm{mM}$ of quinine, which inhibits the mechano-electrical transducer channel, inhibited the influx of $\mathrm{Tl}^{+}$into the hair cells of wild-type cochlea. (d) Changes in fluorescence intensity before and $120 \mathrm{~s}$ after the addition of thallium in the contralateral cochleae of uninjected and AAV-injected mice. The yellow dotted line shows the boundary between the inner (IHCs) and outer hair cells (OHCs). Scale bar, $50 \mu \mathrm{m}$. (e) Sequential changes in $\mathrm{F} / \mathrm{F}$ values after Tl ${ }^{+}$stimulus in the IHCs and OHCs of the cochleae of wild-type, AAV-injected mutant, and uninjected mutant mice. (f) Comparative analysis of the change in the slope of the fluorescence density of the IHCs and OHCs of wild-type, injected mutant, and uninjected mutant mice. $* * * * p<0.001$. 


\section{In vivo gene editing of KCNQ4 variants linked to DFNA2}

To investigate the clinical feasibility of in vivo gene editing to reverse the hearing loss caused by variants in $K C N Q 4$, we searched all the variants in $K C N Q 4$ associated with DFNA2 from the

databases, including the Human Gene Mutation Database (HGMD professional v2020.4) and ClinVar. We found 49 mutations in $K C N Q 4$, including frameshift and splicing variants, and then analyzed the best gene editing scores for each mutation using the deepSpCas9 prediction program with various combinations of sgRNA and Cas9 variants ${ }^{33}$. We found that the gene editing scores for most mutations were comparable to or even higher than those for $\mathrm{p}$.W276S (Fig. 6 and Supplementary Table 3). In particular, wild-type SpCas9 was the most efficient for all mutations in $K C N Q 4$. This indicates that the majority of variants in KCNQ4 may be potential targets for in vivo gene editing. 
Fig. 6

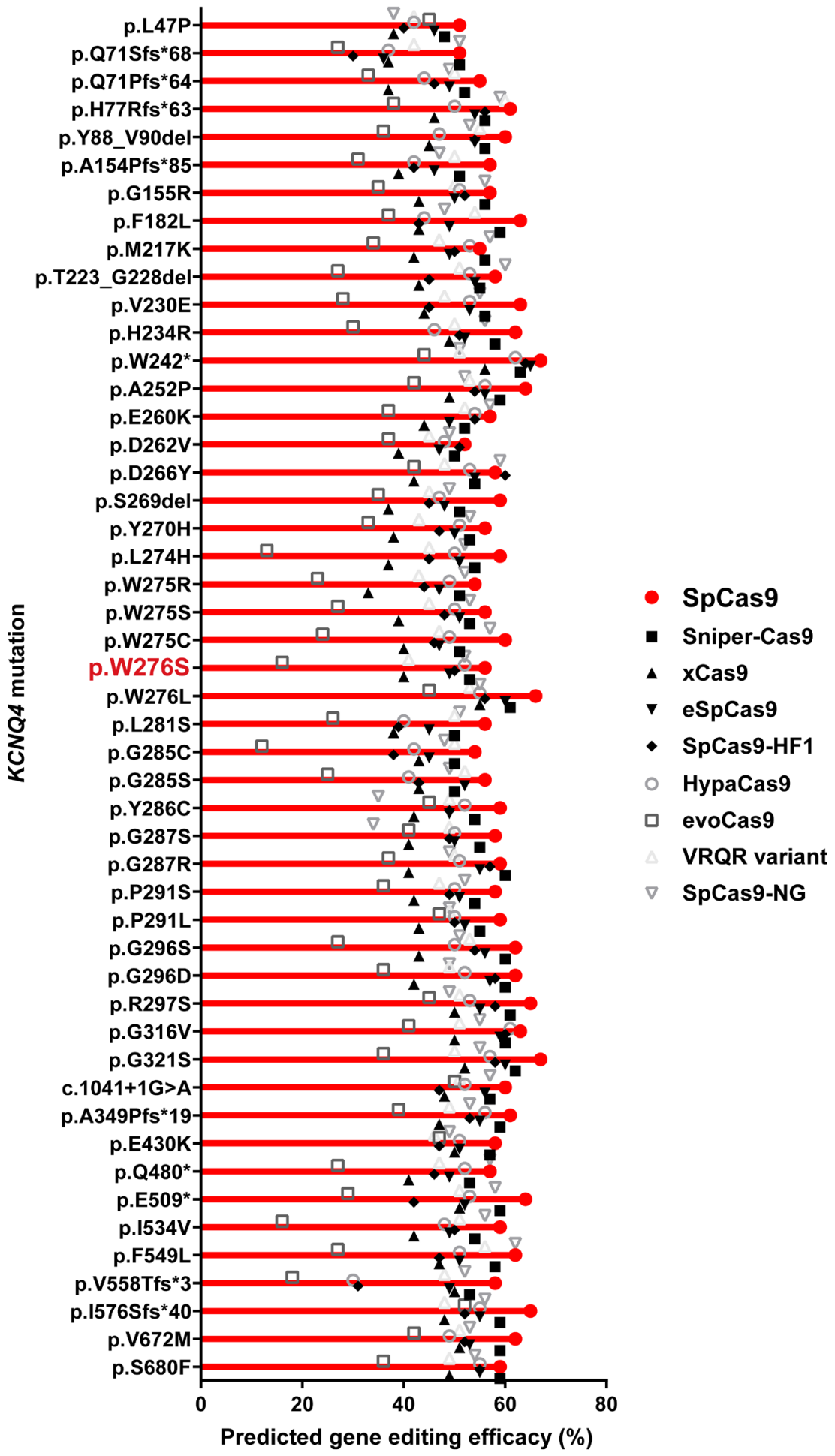

Fig. 6. In vivo gene editing scores for mutations in $K C N Q 4$. In total, 49 mutations linked to DFNA2 were selected to evaluate the in vivo gene editing scores. Missense and frameshift mutations, as well as splicing variants, were included. The DeepSpCas9 prediction program was utilized to test the predicted efficiency of in vivo gene editing based on combinations of candidate sgRNAs for KCNQ4 mutant sequences and Cas9 variant forms. 


\section{Discussion}

In the present study, pathogenic $K C N Q 4$ variants were the most commonly identified in patients with AHL. We successfully applied in vivo gene editing with Cas9 nuclease to ameliorate progressive hearing loss in the dominant-negative Kcnq $4^{\mathrm{W} 276 \mathrm{~S} /+}$ murine model. Moreover, we revealed that even minimal gene editing efficiency can restore the hyperpolarized steady-state of OHCs in the cochlea.

While in vivo genome editing can remedy an extensive range of genetic diseases, the cochlea-targeting capabilities need to be further improved. Compared with delivery to organs such as the liver, muscle, brain, and eye, the delivery of gene-editing-related materials to the cochlea is very difficult ${ }^{40}$ as the cochlea is surrounded by a hard cortical bone and bloodlabyrinth barrier that hinders efficient delivery. However, our results indicate that strong gene editing efficiency is not necessarily required to sufficiently restore hearing in the Kcnq4 ${ }^{\mathrm{W} 276 \mathrm{~S} /+}$ murine model; specifically, $\sim 0.6 \%$ of gene editing efficiency at the genomic DNA level sufficiently rescued the auditory phenotype with dual AAV plasmids with split SpCas9 and sgRNA. These data are consistent with those of a previous study, which reported sufficient hearing restoration when the in vivo gene editing efficiency was $\sim 0.6 \%$ in Beethoven mice harboring the Tmcl mutant allele ${ }^{41}$. The discrepancy between the efficiency and phenotypic rescue can be explained by several technical reasons. For instance, following the preparation of cochlear samples for sequencing, the actual number of inactivated mutant alleles may be inevitably underestimated in some cases. In addition, genetic mosaicism arising from CRISPRCas9-mediated non-homologous end joining (NHEJ) and homology-directed repair is often observed in vitro and in vivo ${ }^{42}$; therefore, the effect of Cas9 on the mutation cut site may be 
compromised. Nevertheless, we consider that the gene editing threshold required for observable hearing restoration in the cochlea is relatively lower than that for other diseases.

KCNQ4 is crucial for the pathogenesis of AHL, as demonstrated by previous studies ${ }^{6}$. Genetic alterations in $\mathrm{KCNQ} 4$ result in the absence of potassium recycling in the $\mathrm{OHCs}$ of the cochlea, a phenomenon that enhances susceptibility to noise and promotes progressive hearing loss. In this respect, genome editing to treat AHL should focus on the OHCs, which are the sites where this sensory organ begins to degenerate. Interestingly, the degeneration of OHCs occurs slowly for decades with onset occurring in older individuals, suggesting that the therapeutic window for treating AHL using gene therapy is adequate for attempting to decelerate progressive hearing $\operatorname{loss}^{26,43,44}$. Given that most mutations related to DFNA2, including indel mutations, can be efficiently edited via SpCas9 and its variant forms (Fig. 6), we theorize that this gene editing strategy, i.e., inactivating $K C N Q 4$ dominant-negative mutant alleles, can be applied to other $K C N Q 4$ variants as well.

Although these data indicate that in vivo gene editing is applicable for treating AHL caused by the degeneration of $\mathrm{OHCs}$, the efficiency of gene editing and the delivery vehicle used should be further improved. The high-fidelity capsid of AAV, which is optimized for targeting the OHCs, will be required to accomplish these goals; this strategy has improved the transduction rate of AAV9-PHP.B capsid into $\mathrm{OHCs}^{41}$, and can therefore be used for further gene editing applications. Moreover, other delivery methods, such as via mRNA and RNP, will be considered to avoid the collateral safety issues associated with administering viral injections to humans. Although RNP injection aids in correcting alleles in the cochlea, as seen in the present and previous studies ${ }^{22}$, the administration of RNP should be more comfortable than performing a cochleostomy, which is still a challenging procedure for many otology surgeons. 
In conclusion, we demonstrated that editing of the Kcnq4 gene in the OHCs-in an effort to enhance the functional channel activity of $\mathrm{KCNQ} 4$ - can sufficiently restore hearing function in a murine model that recapitulated DFNA2, even at low gene editing efficiencies. Our findings provide a rationale for the clinical application of gene editing to treat AHL.

\section{Methods}

\section{Genetic diagnosis in patients with $A H L$}

This study was approved by the institutional review board of the Severance Hospital, Yonsei University Health System (Seoul, Republic of Korea) (IRB \#4-2015-0659). We obtained informed written consent from individuals with hearing loss for participation in this study and publication of their clinical data. In total, 213 unrelated individuals with AHL were enrolled. Based on the auditory threshold (referred to as PTA4: the average threshold at 500, 1000, 2000, and $4000 \mathrm{~Hz}$ ) in a pure-tone audiogram, AHL was determined based on a cut-off value of $\mathrm{PTA}_{4}$ $>40 \mathrm{~dB}$ and adult-onset $>$ late second decade. For molecular genetic testing, genomic DNA was extracted for whole exome or targeted 207 deafness genes. For variant filtering and interpretation process of mutation identification, the causative genes and mutations for hearing loss were analyzed based on a literature review and database search including the hereditary hearing loss homepage (https://hereditaryhearingloss.org/), Deafness Variation Database

(http://deafnessvariationdatabase.org/), HGMD (professional v2020.4), ClinVar (https://www.ncbi.nlm.nih.gov/clinvar/) and the Online Mendelian Inheritance in Man (OMIM) database (http://www.ncbi.nlm.nih.gov/omim) ${ }^{43}$.

Generation of the Kcnq4 p.W276S mouse model 
Animal experiment protocols were reviewed and approved by the Institutional Animal Care and Use Committee of the Yonsei University College of Medicine (\#2017-0295). Kcnq4 W276S knock-in mice were generated by Macrogen, Inc. (Republic of Korea). Briefly, C57BL/6N female mice were treated with mare serum gonadotropin (7.5 IU) and human chorionic gonadotropin (5 IU). After $48 \mathrm{~h}$, the mice were allowed to mate with C57BL/6N stud male mice. The next day, female mice with vaginal plugs were euthanized, and the fertilized embryos were harvested. A mixture of sgRNA and Cas9 protein was microinjected into one-cell embryos incubated at $37{ }^{\circ} \mathrm{C}$ for $1-2 \mathrm{~h}$. The injected one-cell staged embryos $(n=14-16)$ were transplanted into the oviducts of pseudopregnant recipient mice (ICR). F0 mice were genotyped by PCR using tail-cut samples (primers: 5'-GCATTCCTAGGGGTCTTTCC-3'; 5'CATCAGGTTCTTGCGAACCT-3') and subjected to the T7E1 assay. T7E1-positive samples underwent TA cloning and were analyzed by Sanger sequencing. In the F1 generation, genotyping was performed using PCR with the same primers, and the PCR products were digested using Nde1 for 2-3 h to identify the two-band pattern in agarose gel electrophoresis.

\section{Plasmid construction}

Cas9 and sgRNAs were expressed using the CMV promoter-driven Cas9-2A-mRFP-2A-Puro plasmid (hereafter, Cas9-puro vector) and the hU6 promoter-driven sgRNA plasmid, respectively. The plasmids were purchased from Toolgen (Seoul, Republic of Korea); the vector maps of these plasmids are shown in Supplementary Fig. 3.

For the AAV experiments, the previously validated expression plasmids were used to express the split C-Intein-C-Cas9 expression plasmid (N-Cas9 N-intein and C-Intein C-Cas9 plasmids, a gift from Oskar Ortiz) ${ }^{45}$. To prepare the sgRNA-N-Cas9-N-intein, we modified the backbone 
plasmid (addgene plasmid \# 60958) generated by Swiech et al. ${ }^{46}$. Briefly, the backbone vector was digested with $\mathrm{XbaI}$ and EcoRI, and the $\mathrm{N}$-Cas9-N-intein fragment digested by $\mathrm{XbaI}$ and EcoRI was ligated to the backbone (addgene plasmid \# 60958) vector to express the U6-sgRNAN-Cas9 N-intein. Finally, to clone the selected sgRNA (sgRNA-T3), the vector was digested with SapI and ligated with annealed oligonucleotides. All plasmid and oligonucleotide sequences are shown in Supplementary Table 4 and Supplementary Note 1.

\section{sgRNA preparation and transfection of reporter cell line}

The sgRNA target sequences were manually designed based on the protospacer-adjacent motif (PAM-NGG) sequences near the mutation target locus (c.830G $>$ C) (Fig. 2a and Supplementary Fig. 2a). The sgRNA-encoding vector (pRG2-sgRNA) was digested with BsaI and ligated with the annealed oligonucleotides; oligonucleotide sequences are listed in Supplementary Table 4. Reporter cells for the Kcnq4 mutant were transfected with plasmid mixtures containing Cas9puro and individual U6-sgRNAs encoding plasmids at a 1:2 weight ratio using Lipofectamine 2000 according to manufacturer's instructions. The cells were harvested $3 \mathrm{~d}$ after transfection and analyzed.

The reporter vector was designed based on a previously reported comparable construct $^{47,48}$. The Lenti-reporter plasmid constitutively expresses $R F P$ and the respective Kcnq4 target sequence containing a c.830G $>\mathrm{C}$ mutation (35 bp in length), along with additional sequences encoding eGFP, positioned out-of-frame relative to RFP (Fig. 2a and Supplementary Fig. 2b). The target sequence contains a 20-bp region with adjacent PAMs for gRNA binding and Cas9-mediated double breaks (Supplementary Fig. 2b). Thus, the recruitment of Cas9 to the respective Kcnq4 mutant-specific target sequence by properly spaced sgRNA results in a 
double-stranded break, leading to an insertion or deletion (indel) mutation at the target locus. Because of the codon triplet, one out of three repairs results in a significant "in frame" fusion of $e G F P$ with $m R F P$ located upstream of the cleavage site.

\section{IVT-sgRNA preparation}

sgRNA was transcribed in vitro using T7 RNA polymerase with templates generated by annealing and the extension of two complementary oligonucleotides (Supplementary Table 4) with a mMESSAGE mMACHINE® T7 Ultra RNA Synthesis kit (\#AM1345; Invitrogen, Carlsbad, CA) according to manufacturer's instructions. RNA was purified using a MEGAclear kit (\#AM1908; Invitrogen). Purified RNA was quantified using a Nanodrop and gel electrophoresis.

\section{Lentivirus production and reporter cell line generation}

Lenti-reporter plasmids containing the Kcnq4 mutation (c.830G $>$ C) were constructed as previously described ${ }^{47,48}$. Briefly, oligonucleotides, including the target sequence (Supplementary Table 4), were synthesized by Macrogen and annealed in vitro using a thermocycler $\left(95{ }^{\circ} \mathrm{C}\right.$ for $5 \mathrm{~min}$ and then ramped down to $25{ }^{\circ} \mathrm{C}$ at $\left.5{ }^{\circ} \mathrm{C} / \mathrm{min}\right)$. The annealed oligonucleotides were ligated into the Lenti-reporter vectors digested with EcoR1 and BamH1.

The lentivirus was produced as previously described $33,49,50$. Briefly, three transfer plasmids, containing the Kcnq4 mutation, psPAX2, and pMD2.G, were mixed at a weight ratio of $4: 3: 1$ to yield the plasmid mixture $(10 \mu \mathrm{g})$. HEK293T cells at $80-90 \%$ confluence were transfected with this mixture using Lipofectamine 2000 (Invitrogen). Viral supernatant was 
harvested 48 and $72 \mathrm{~h}$ post-transfection, filtered through a Millex-HV $0.45-\mu \mathrm{m}$ low-proteinbinding membrane (Millipore, Darmstadt, Germany), and concentrated by ultracentrifugation.

Next, a reporter cell line was generated as previously described ${ }^{33,49,50}$. Briefly, HEK293T cells $\left(2.2 \times 10^{6}\right)$ were seeded in a $100-\mathrm{mm}$ cell culture dish and incubated overnight. Cells were transduced with the lentiviral reporter virus at MOI 0.1 with polybrene $(4 \mu \mathrm{g} / \mathrm{mL})$ and incubated for $15-18 \mathrm{~h}$. Untransduced cells were removed by zeocin ( $2 \mu \mathrm{g} / \mathrm{mL}$; InvivoGen) treatment from 3 to $5 \mathrm{~d}$. When almost all untransduced cells had died and the surviving cells had expanded, cells were maintained in zeocin $(2 \mu \mathrm{g} / \mathrm{mL})$ for further use as previously described ${ }^{51}$. The reporter cell line expressing Kcnq4 with the appropriate SpCas9 and sgRNA expression plasmids was transfected. The activity of each sgRNA was detected 72-h post-transfection.

\section{Cell culture}

Human embryonic kidney 293T (HEK293T) cells were purchased from the American Type Culture Collection (ATCC; Manassas, VA). HEK293T, reporter cells, and Neuro2A cells were maintained in Dulbecco's modified Eagle medium (DMEM; Invitrogen) supplemented with 100 $\mathrm{U} / \mathrm{mL}$ penicillin, $100 \mu \mathrm{g} / \mathrm{mL}$ streptomycin, and $10 \%$ fetal bovine serum.

\section{T7E1 assay}

T7E1 assay was performed as previously described ${ }^{52,53}$. Briefly, the target site was amplified using nested PCR with the appropriate primers (Supplementary Table 4). Amplicons were denatured by heating and annealed to allow the formation of heteroduplex DNA and treated with T7 endonuclease 1 ( $5 \mathrm{U}$; New England Biolabs) for $20 \mathrm{~min}$ at $37^{\circ} \mathrm{C}$, followed by electrophoresis on a $2 \%$ agarose gel. Mutation frequencies were calculated as previously described based on the 
band intensities using Image J and the following equation ${ }^{54}$ : mutation frequency $(\%)=100 \times(1-$ $\left.(1-\text { fraction cleaved })^{1 / 2}\right)$, where the fraction cleaved is the total relative density of cleavage bands divided by the sum of the relative density of the cleavage bands and uncut bands.

\section{DNA extraction and targeted deep sequencing}

The extracted genomic DNA of HEK293T cells was isolated using the Wizard Genomic DNA Purification Kit (Promega, Madison, WI) according to manufacturer's instructions. To isolate mouse genomic DNA, DNA was extracted using an Exgene Tissue SV Kit (GeneAll, Seoul, Republic of Korea) after surgical dissociation of the cochlear tissue. The on-target region within genomic DNA was amplified using eTaq or Pfu DNA polymerase (Promega). Equal amounts of PCR amplicons were subjected to paired-end read sequencing using Illumina MiSeq at Bio Medical Laboratories (Minworth, UK). The region, including the target sites, was amplified by nested PCR using the appropriate primers (Supplementary Table 4). Indels mapped around the Cas9 nuclease cleavage site ( 3 bp upstream of the PAM) were considered the result of nucleaseinduced NHEJ-mediated mutagenesis.

\section{Allele-specific indel analysis}

To analyze the genotype correction rate by CRISPR on mutant alleles, allele-specific indel analysis strategies were utilized. Gene editing efficiency of mutant alleles, rather than that of wild-type alleles, was prioritized. Because indels at the mutation site can hinder identification of the origin of the edited allele (i.e., whether the wild-type or mutant allele was edited), the mutant allele was identified using the synonymous variant c.810C $>$ A (Fig. 1b) located 20-base-pairs upstream of the mutation spot; we only counted the reads with synonymous variants. Reads with 
both the synonymous variant and any indel pattern ranging from 1 to $8 \mathrm{bp}$ were regarded as edited mutant alleles, ruling out sequencing errors. Furthermore, the gene editing efficiencies of the injected and uninjected cochleae from the same mouse were compared to minimize interindividual variations. Fold change in gene editing per mouse was calculated as the mutant allele indel rate of injected cochlea divided by that of uninjected cochlea in the same mouse.

\section{AAV vector generation}

Plasmids of split Cas9 (i.e., C-Cas9 and N-Cas9) and gRNA were packaged into AAV/Anc80 using the Harvard vector core ${ }^{34}$. AAV titers were validated using qRT-PCR targeting the inverted terminal repeat of the virus (Supplementary Fig. 3e). AAV stock concentrations were $1.29 \times 10^{12}$ and $4.18 \times 10^{12}$ genome copies/mL for C-Cas9 and N-Cas9 with sgRNA, respectively. The final injected AAV titer was estimated to be $1.00 \times 10^{9}$ genome copies $/ 1 \mu \mathrm{L}$ in one cochlea.

\section{Optimization of RNP injection material using in vitro and explant samples}

To identify the optimal injection-mixture ratio and incubation times, three injection routes were evaluated as described previously with slight modifications (Supplementary Fig. 4a). Purified Cas9 proteins were mixed with sgRNA in an optimized ratio and incubated at $25^{\circ} \mathrm{C}$ for 5 min, followed by Lipofectamine 2000 addition. The sample was incubated for 20 min before injection into the cochlea. The optimized ratio was determined after comparing the NGS indel rates of four different mixtures of Cas9, sgRNA, and Lipofectamine 2000 reagents. Although the in vivo conditions are completely different from in vitro and explant culture system conditions, the optimal ratio of injection material mixture may not be markedly different between both 
conditions. Ex vivo gene editing efficiencies varied among the different combinations (from $26.1 \%$ to $32.2 \%$; Supplementary Fig. 4b). As the practically available volume of injection materials into the cochlea of the P2-P5 pup is within $1 \mu \mathrm{L}$, the sgRNA (1.0 $\mu \mathrm{g})$ and Cas9 (1.5 $\mu \mathrm{g})$ concentrations were maximized.

\section{Inner ear injection}

AAV virus or the RNP complex were injected into the inner ear of Kcnq4 W276S heterozygous mutant pups at P1-P3. After the pups were anesthetized by exposure to ice for 2 min (hypothermia), a post-auricular incision was made to expose the injection route. Using a glass pipette and a Nanoliter2020 Injector (World Precision Instruments, Hertfordshire, UK), the injection material $(1 \mu \mathrm{L})$ was delivered into cochlea at a consistent rate of $40 \mathrm{~nL} / \mathrm{min}$. After injection, a suture was made to close the cut skin. The pup was placed on a heating pad to recover for at least $5 \mathrm{~min}$. Various injection routes were comprehensively tested in more than 500 pups to minimize the physical damage to the cochlea during the injection.

\section{Hearing test including $A B R$ and $D P O A E$}

ABR thresholds were measured in a sound-proof chamber using Tucker-Davis Technologies (TDT) RZ6 digital signal processing hardware and BioSigRZ software (Alachua, FL, USA). Sub-dermal needles (electrodes) were positioned at the vertex and ventrolateral to the right and left ears of anesthetized mice. A calibrated click stimulus (10- $\mu$ s duration) or tone burst stimuli (5-ms duration) were produced at $6,12,18,24$, and $30 \mathrm{kHz}$ using the SigGenRZ software and an RZ6 digital signal processor and delivered to the ear canal using a multi-field 1 (MF1) magnetic speaker (TDT). The stimulus intensity was increased from 10 to $90 \mathrm{~dB}$ SPL in 5-dB steps. ABR 
signals were fed into a low-impedance Medusa Biological Amplifier System (RA4LI, TDT), which delivered the signal to the RZ6 digital signal processing hardware. The recorded signals were filtered using a $0.5-1 \mathrm{kHz}$ band-pass filter, and ABR waveforms in response to 256-tone bursts were averaged. ABR thresholds for each frequency were determined using the BioSigRZ software.

For DPOAE, a combination TDT microphone-speaker system was utilized. Primary stimulus tones were produced using an RZ6 digital signal processor with the SigGenRZ software and delivered using a custom probe with an ER 10B+ microphone (Etymotic, Elk Grove Village, IL) and MF1 speakers positioned in the ear canal. Primary tones were set at a frequency ratio (f2/f1) of 1.2 with target frequencies of $6,12,16,18,22,24$, and $30 \mathrm{kHz}$. The $\mathrm{f} 2$ intensity levels were the same as the $\mathrm{f} 1$ intensity level $(\mathrm{L} 1=\mathrm{L} 2)$. Sounds caused by the primary tones were received by the ER 10B+ microphone and recorded using the RZ6 digital signal processor. The DPOAE input/output (I/O) functions were determined at specific frequencies (6 and $30 \mathrm{kHz})$ with a frequency ratio (f2/f1) of 1.2 and equal intensity levels $(\mathrm{L} 1=\mathrm{L} 2)$. Intensity levels of the primary tones were increased from 20 to $80 \mathrm{~dB}$ SPL in 5-dB SPL increments. Fast Fourier transform (FFT) was performed at each primary tone for the DP grams and at each intensity for the I/O functions using BioSigRZ to determine the average spectra of the two primaries, the $2 \mathrm{f1}$ f2 distortion products, and the noise floors.

\section{Immunohistochemistry and histology}

Immunoblotting and immunofluorescence were performed as previously described ${ }^{55,56}$. Injected and uninjected cochleae were excised after $\mathrm{CO}_{2}$-inhalation-induced euthanization. Temporal bones were fixed overnight in $4 \%$ paraformaldehyde at $4{ }^{\circ} \mathrm{C}$ and decalcified in $120 \mathrm{mM}$ EDTA 
for at least 1 week. Cochleae were dissected in pieces from the decalcified tissue for wholemount immunofluorescence. Tissues were permeabilized with $0.3 \%$ Triton X-100 and blocked with $10 \%$ donkey serum for $1 \mathrm{~h}$ before overnight incubation at $4{ }^{\circ} \mathrm{C}$ with the following primary antibodies: mouse anti-Tuj1, purified anti-tubulin beta 3, (1:300;801202; BioLegend), and chicken anti-neurofilament $\mathrm{H}$ antibodies (1:1,000; AB5539; Merck Millipore). DAPI (1:5,000; Invitrogen) was used for nuclear staining $\left(25^{\circ} \mathrm{C}\right.$ for $\left.10 \mathrm{~min}\right)$. For counting IHCs and OHCs, FITC-conjugated phalloidin (1:200; P5282; Sigma-Aldrich) was used to stain F-actin at $25^{\circ} \mathrm{C}$ (15 min). Next, after three rinses with $0.3 \%$ Triton $\mathrm{X}-100$ in $1 \mathrm{X}$ PBS, the specimens were incubated for $1 \mathrm{~h}$ with the secondary antibodies donkey anti-mouse Alexa Fluor 488 (A21202; 1:1,000; Invitrogen) and goat anti-chicken Alexa Fluor 568 (A11041; 1:1,000; Invitrogen). Specimens were mounted in Fluoromount ${ }^{\mathrm{TM}}$ Aqueous Mounting Medium (F4680-25ML, SigmaAldrich) before imaging with a confocal microscope (LSM700; Zeiss, Jena, Germany) with 10×, $20 \times$, or $40 \times$ water-immersion lens, with or without digital zoom, and processed using ZEN software. For IHC and OHC counting, DAPI-positive and Tuj1-positive spiral ganglion neuron (SGN) counting and quantification of the density of the neurofilament heavy chains, $z$-stacks were acquired by the maximum intensity projections of $z$-stacks for each segment by ImageJ. Composite images showing the whole cochlea were constructed in Adobe Photoshop CS3 to display the entire turn of the cochlea. A frequency map of each specimen was drawn using ImageJ based on a previous report ${ }^{57}$. Phalloidin- and DAPI-positive IHCs and OHCs were counted in cochlear regions responsive to different sound frequencies, and segments containing dissection-related damage were omitted from the analysis. For SGN counting, fluorescence intensity of neurofilament heavy chains was quantified at the inner spiral plexus (spiraling 
underneath the IHCs) and unmyelinated outer spiral fibers (spiraling underneath the OHCs) using the auto-threshold algorithm ( "Otsu" method)

\section{Cochlea live-imaging with thallium assay}

The thallium flux assay was performed ex vivo to confirm potassium-induced physiological changes in the hair cells of AAV-injected and uninjected cochleae ${ }^{59-61}$. Briefly, 7-week-old mice with confirmed $\mathrm{ABR}$-induced hearing improvement were euthanized with $\mathrm{CO}_{2}$ to harvest both cochleae (i.e., AAV-injected and contralateral uninjected inner ears). The inner ear of agematched wild-type mice was used as control. The apical bone capsule of the inner ear was removed in cold HBSS with forceps. Each cochlea extracted from the inner ear was fixed with a needle on a confocal glass bottom dish (100350; SPL). FluxOR ${ }^{\mathrm{TM}}$ Potassium Ion Channel Assay (F10016; Invitrogen) was performed according to manufacturer's instructions. The cochlea was incubated ex vivo with FluxOR ${ }^{\mathrm{TM}}$, a fluorescent dye (at $488 \mathrm{~mm}$ excitation wavelength) exclusively sensitive to thallium ions ${ }^{62}$. To confirm whether the FluxOR dye loaded inside the hair cells binds to extracellular $\mathrm{Tl}+$ from the MET channel and increases the fluorescence density, wild-type cochleae treated with quinine $(10 \mathrm{mM}$; an MET channel inhibitor) were used as negative control ${ }^{63}$. After loading the FluxOR dye, it was replaced with assay buffer $(100 \mu \mathrm{L})$, and live-cell recording was performed using confocal microscopy and MetaFluor software. When the baseline was stabilized, stimulus buffer ( $20 \mu \mathrm{L}$ at a final concentration of $2 \mathrm{mM} \mathrm{Tl}^{+}$) was added, and recording was performed for $120 \mathrm{~s}$. After adding the stimulus buffer, $\triangle \mathrm{F} 120$ was calculated relative to the baseline.

\section{KCNQ4 mutation collection and gene editing efficiency prediction}


For collecting all reported $K C N Q 4$ mutations responsible for AHL in patients with deafness, we identified all KCNQ4 variants annotated as "pathogenic/likely pathogenic" in the ClinVar database and/or as "disease-causing mutations (DM)" in the HGMD database (professional v.2020.4). For a total of 49 pathogenic mutations in KCNQ4, combinations of sgRNAs and Cas9 variants were tested for gene editing efficiency in mutant alleles using our algorithm ${ }^{50}$. The best gene editing efficiency for each mutation was compared across all mutations and Cas9 variant types.

\section{Statistical analysis}

Data were pooled from at least three independent experiments and expressed as the mean \pm SD. Differences between two groups were determined using Student's $t$-test or two-way analysis of variance (ANOVA) with Bonferroni's corrections for multiple comparisons and conducted using GraphPad Prism v8.0 (GraphPad Software, San Diego, CA). Significant differences among the three groups were analyzed using one-way ANOVA with Tukey's multiple comparisons test for parametric comparisons and Kruskal-Wallis test with Dunn's multiple comparisons test for nonparametric comparisons. $p<0.05$ was considered significant.

\section{Data and software availability}

The data and codes associated with this study are available from the corresponding author upon reasonable request. The published article includes all datasets/codes generated or analyzed during this study. Deep sequencing data have been deposited in the NCBI Sequence Read Archive (PRJNA691110). 


\section{References}

1. Morton, C.C. \& Nance, W.E. Newborn hearing screening--a silent revolution. N Engl J Med 354, 2151-2164 (2006).

2. Kral, A. \& O'Donoghue, G.M. Profound deafness in childhood. N Engl J Med 363, 1438-1450 (2010).

3. Fortnum, H.M., Summerfield, A.Q., Marshall, D.H., Davis, A.C. \& Bamford, J.M. Prevalence of permanent childhood hearing impairment in the United Kingdom and implications for universal neonatal hearing screening: questionnaire based ascertainment study. Bmj 323, 536-540 (2001).

4. Kidd lii, A.R. \& Bao, J. Recent advances in the study of age-related hearing loss: a mini-review. Gerontology 58, 490-496 (2012).

5. Fransen, E. et al. Occupational noise, smoking, and a high body mass index are risk factors for age-related hearing impairment and moderate alcohol consumption is protective: a European population-based multicenter study. J Assoc Res Otolaryngol 9, 264-276; discussion 261-263 (2008).

6. Peixoto Pinheiro, B. et al. Age-related hearing loss pertaining to potassium ion channels in the cochlea and auditory pathway. Pflugers Arch (2020).

7. Hong, J.W. et al. The prevalence and factors associated with hearing impairment in the Korean adults: the 2010-2012 Korea National Health and Nutrition Examination Survey (observational study). Medicine (Baltimore) 94, e611 (2015).

8. Van Eyken, E. et al. KCNQ4: a gene for age-related hearing impairment? Hum Mutat 27, 10071016 (2006).

9. Wu, P.Z., O'Malley, J.T., de Gruttola, V. \& Liberman, M.C. Age-Related Hearing Loss Is Dominated by Damage to Inner Ear Sensory Cells, Not the Cellular Battery That Powers Them. $J$ Neurosci 40, 6357-6366 (2020).

10. $\mathrm{Li}, \mathrm{H}$. et al. Applications of genome editing technology in the targeted therapy of human diseases: mechanisms, advances and prospects. Signal Transduct Target Ther 5, 1 (2020).

11. Anzalone, A.V., Koblan, L.W. \& Liu, D.R. Genome editing with CRISPR-Cas nucleases, base editors, transposases and prime editors. Nat Biotechnol 38, 824-844 (2020).

12. Akil, O. et al. Dual AAV-mediated gene therapy restores hearing in a DFNB9 mouse model. Proc Natl Acad Sci U S A 116, 4496-4501 (2019).

13. Shibata, S.B. et al. RNA Interference Prevents Autosomal-Dominant Hearing Loss. Am J Hum Genet 98, 1101-1113 (2016).

14. Taiber, S. et al. Neonatal AAV gene therapy rescues hearing in a mouse model of SYNE4 deafness. EMBO Mol Med, e13259 (2020).

15. Askew, C. et al. Tmc gene therapy restores auditory function in deaf mice. Sci Transl Med 7, 295ra108 (2015).

16. Isgrig, K. et al. Gene Therapy Restores Balance and Auditory Functions in a Mouse Model of Usher Syndrome. Mol Ther 25, 780-791 (2017).

17. Pan, B. et al. Gene therapy restores auditory and vestibular function in a mouse model of Usher syndrome type 1c. Nat Biotechnol 35, 264-272 (2017).

18. Lentz, J.J. et al. Rescue of hearing and vestibular function by antisense oligonucleotides in a mouse model of human deafness. Nat Med 19, 345-350 (2013).

19. Akil, O. et al. Restoration of hearing in the VGLUT3 knockout mouse using virally mediated gene therapy. Neuron 75, 283-293 (2012).

20. Ding, N., Lee, S., Lieber-Kotz, M., Yang, J. \& Gao, X. Advances in genome editing for genetic hearing loss. Adv Drug Deliv Rev (2020).

21. Géléoc, G.G.S. \& El-Amraoui, A. Disease mechanisms and gene therapy for Usher syndrome. Hear Res 394, 107932 (2020).

22. Gao, X. et al. Treatment of autosomal dominant hearing loss by in vivo delivery of genome editing agents. Nature 553, 217-221 (2018).

23. Gyorgy, B. et al. Allele-specific gene editing prevents deafness in a model of dominant progressive hearing loss. Nat Med 25, 1123-1130 (2019).

24. Yeh, W.H. et al. In vivo base editing restores sensory transduction and transiently improves auditory function in a mouse model of recessive deafness. Sci Transl Med 12 (2020). 
25. Moser, T. Gene therapy for deafness: How close are we? Sci Transl Med 7, 295fs228 (2015).

26. Jung, J. et al. Rare KCNQ4 variants found in public databases underlie impaired channel activity that may contribute to hearing impairment. Exp Mol Med 51, 1-12 (2019).

27. Naito, T. et al. Comprehensive genetic screening of KCNQ4 in a large autosomal dominant nonsyndromic hearing loss cohort: genotype-phenotype correlations and a founder mutation. PLoS One 8, e63231 (2013).

28. Van Laer, L. et al. The contribution of genes involved in potassium-recycling in the inner ear to noise-induced hearing loss. Hum Mutat 27, 786-795 (2006).

29. Kubisch, C. et al. KCNQ4, a novel potassium channel expressed in sensory outer hair cells, is mutated in dominant deafness. Cell 96, 437-446 (1999).

30. Coucke, P.J. et al. Mutations in the KCNQ4 gene are responsible for autosomal dominant deafness in four DFNA2 families. Human molecular genetics 8, 1321-1328 (1999).

31. Akita, J., Abe, S., Shinkawa, H., Kimberling, W.J. \& Usami, S. Clinical and genetic features of nonsyndromic autosomal dominant sensorineural hearing loss: KCNQ4 is a gene responsible in Japanese. J Hum Genet 46, 355-361 (2001).

32. Van Camp, G. et al. A mutational hot spot in the KCNQ4 gene responsible for autosomal dominant hearing impairment. Hum Mutat 20, 15-19 (2002).

33. Kim, H.K. et al. Deep learning improves prediction of CRISPR-Cpf1 guide RNA activity. Nat Biotechnol 36, 239-241 (2018).

34. Landegger, L.D. et al. A synthetic AAV vector enables safe and efficient gene transfer to the mammalian inner ear. Nat Biotechnol 35, 280-284 (2017).

35. Pan, B. et al. Gene therapy restores auditory and vestibular function in a mouse model of Usher syndrome type 1c. Nat Biotechnol 35, 264-272 (2017).

36. Lee, S., Dondzillo, A., Gubbels, S.P. \& Raphael, Y. Practical aspects of inner ear gene delivery for research and clinical applications. Hear Res 394, 107934 (2020).

37. Liu, S. et al. TMC1 is an essential component of a leak channel that modulates tonotopy and excitability of auditory hair cells in mice. Elife 8 (2019).

38. Corey, D.P. \& Holt, J.R. Are TMCs the Mechanotransduction Channels of Vertebrate Hair Cells? J Neurosci 36, 10921-10926 (2016).

39. Housley, G.D. \& Ashmore, J.F. lonic currents of outer hair cells isolated from the guinea-pig cochlea. J Physiol 448, 73-98 (1992).

40. van Haasteren, J., Li, J., Scheideler, O.J., Murthy, N. \& Schaffer, D.V. The delivery challenge: fulfilling the promise of therapeutic genome editing. Nat Biotechno/ 38, 845-855 (2020).

41. Wu, J. et al. Single and Dual Vector Gene Therapy with AAV9-PHP.B Rescues Hearing in Tmc1 Mutant Mice. Mol Ther (2020).

42. Wolf, D.P., Mitalipov, P.A. \& Mitalipov, S.M. Principles of and strategies for germline gene therapy. Nat Med 25, 890-897 (2019).

43. Jung, J. et al. Whole-exome sequencing identifies two novel mutations in KCNQ4 in individuals with nonsyndromic hearing loss. Sci Rep 8, 16659 (2018).

44. Shin, D.H. et al. A recurrent mutation in KCNQ4 in Korean families with nonsyndromic hearing loss and rescue of the channel activity by KCNQ activators. Hum Mutat 40, 335-346 (2019).

45. Truong, D.J. et al. Development of an intein-mediated split-Cas9 system for gene therapy. Nucleic Acids Res 43, 6450-6458 (2015).

46. Swiech, L. et al. In vivo interrogation of gene function in the mammalian brain using CRISPRCas9. Nat Biotechno/ 33, 102-106 (2015).

47. Kim, H. et al. Surrogate reporters for enrichment of cells with nuclease-induced mutations. Nat Methods 8, 941-943 (2011).

48. Ramakrishna, S. et al. Gene disruption by cell-penetrating peptide-mediated delivery of Cas9 protein and guide RNA. Genome Res 24, 1020-1027 (2014).

49. Kim, H.K. et al. In vivo high-throughput profiling of CRISPR-Cpf1 activity. Nat Methods 14, 153159 (2017).

50. Kim, H.K. et al. SpCas9 activity prediction by DeepSpCas9, a deep learning-based model with high generalization performance. Sci Adv 5, eaax9249 (2019).

51. Shalem, O. et al. Genome-scale CRISPR-Cas9 knockout screening in human cells. Science 343, 84-87 (2014). 
52. Kim, Y.H., Ramakrishna, S., Kim, H. \& Kim, J.S. Enrichment of cells with TALEN-induced mutations using surrogate reporters. Methods 69, 108-117 (2014).

53. Kim, H.J., Lee, H.J., Kim, H., Cho, S.W. \& Kim, J.S. Targeted genome editing in human cells with zinc finger nucleases constructed via modular assembly. Genome Res 19, 1279-1288 (2009).

54. Guschin, D.Y. et al. A rapid and general assay for monitoring endogenous gene modification. Methods Mol Biol 649, 247-256 (2010).

55. Gee, H.Y., Tang, B.L., Kim, K.H. \& Lee, M.G. Syntaxin 16 binds to cystic fibrosis transmembrane conductance regulator and regulates its membrane trafficking in epithelial cells. J Biol Chem 285, 35519-35527 (2010).

56. Son, E.J. et al. Conserved role of Sonic Hedgehog in tonotopic organization of the avian basilar papilla and mammalian cochlea. Proc Natl Acad Sci U S A 112, 3746-3751 (2015).

57. Sanz, L. et al. Swept-sine noise-induced damage as a hearing loss model for preclinical assays. Front Aging Neurosci 7, 7 (2015).

58. Wu, P.-Z. et al. Primary Neural Degeneration in the Human Cochlea: Evidence for Hidden Hearing Loss in the Aging Ear. Neuroscience 407 (2018).

59. Weaver, C.D., Harden, D., Dworetzky, S.I., Robertson, B. \& Knox, R.J. A thallium-sensitive, fluorescence-based assay for detecting and characterizing potassium channel modulators in mammalian cells. J Biomol Screen 9, 671-677 (2004).

60. Weaver, C.D. Thallium Flux Assay for Measuring the Activity of Monovalent Cation Channels and Transporters. Methods Mol Biol 1684, 105-114 (2018).

61. Yu, H.B., Li, M., Wang, W.P. \& Wang, X.L. High throughput screening technologies for ion channels. Acta Pharmacol Sin 37, 34-43 (2016).

62. Beacham, D.W., Blackmer, T., M, O.G. \& Hanson, G.T. Cell-based potassium ion channel screening using the FluxOR assay. J Biomol Screen 15, 441-446 (2010).

63. Alharazneh, A. et al. Functional hair cell mechanotransducer channels are required for aminoglycoside ototoxicity. PLoS One 6, e22347 (2011). 


\section{Acknowledgments}

We sincerely thank our great mentor, Prof. Won Sang Lee, for his devotion and unwavering support to establish the Precision Medicine Center for Yonsei Ear Science. This study was supported by the Basic Science Research Program of the National Research Foundation of Korea (grant numbers 2018R1A5A2025079 to H.Y.G and H.H.K., 2020R1A2C3005787 to C.J.Y., and 2019R1A2C1084033 to J.J.) and the Korean Health Technology R\&D Project of the Ministry of Health and Welfare, Republic of Korea (grant number HI17C0676 to H.H.K.).

\section{Author contributions}

J.J., J.Y.C., H.Y.G., and H.H.K. conceived and designed the study; B.W.N., J.H.R., R.G., H.L., and K.M.K. performed the experiments; B.W.N. and J.H.R. performed data analysis and contributed to data interpretation; R.G. analyzed the efficiency of gene editing materials and performed in vitro validation; B.W.N., J.H.R., R.G., and J.J. wrote the manuscript. All the authors revised the manuscript and approved the final version for publication.

\section{Declaration of interests}

The authors declare no competing interests. 
a

Adult-onset hearing loss $(n=213)$

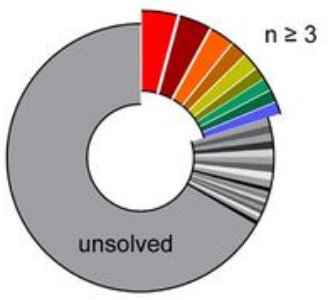

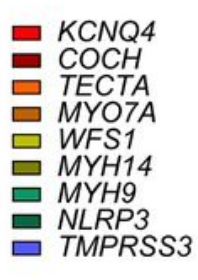

b

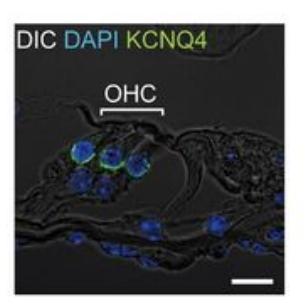

C

\section{Kcnq4}

GRCm39/mm39; chr4:120572970 C> G

NM_001081142 c.830G>C (exon5)

$$
\begin{array}{ll}
\text { c. } 810 \mathrm{C}>\mathrm{A} ; & \text { c. } 830 \mathrm{G}>\mathrm{C} ; \\
\text { p.S269 }= & \text { p.W276S }
\end{array}
$$

GACTTCTCCTCATATGCCGACTCGCTCTGGTCGGGGACGGTG Ndel re-cut prevention d

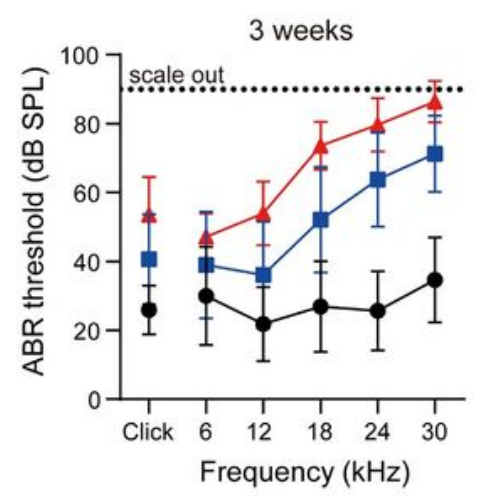

e

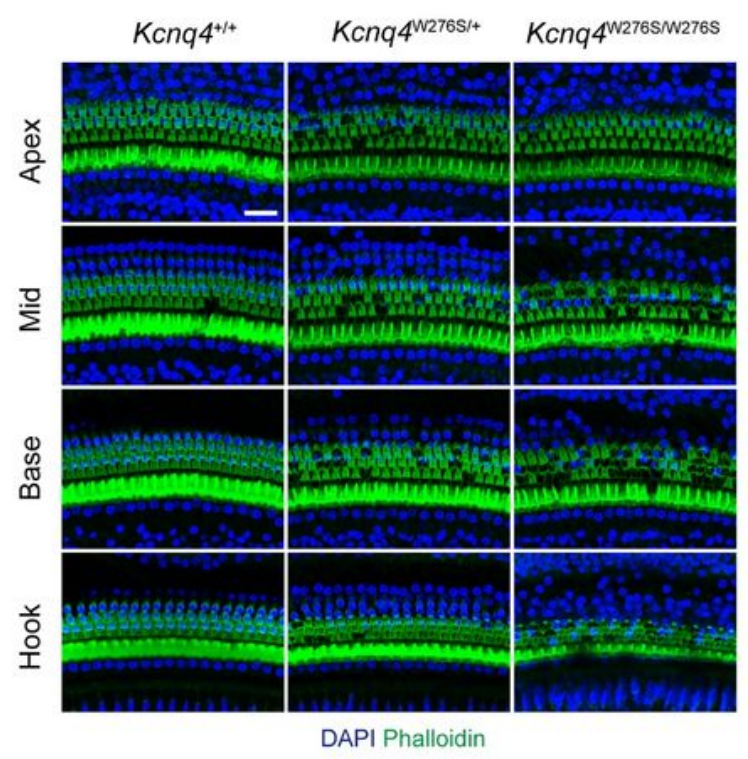

7 weeks

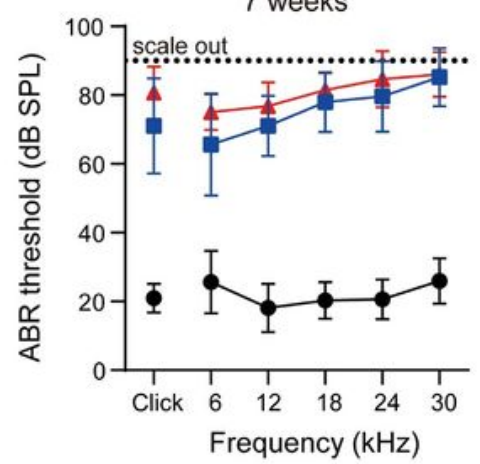

11 weeks

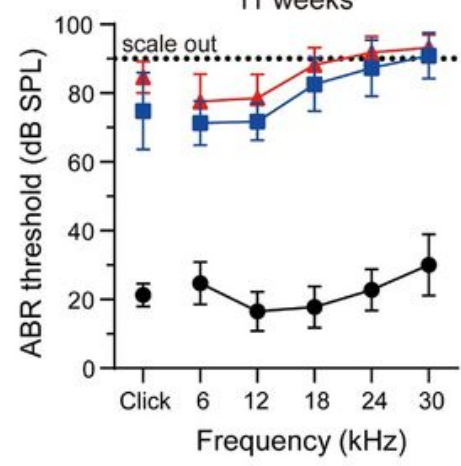

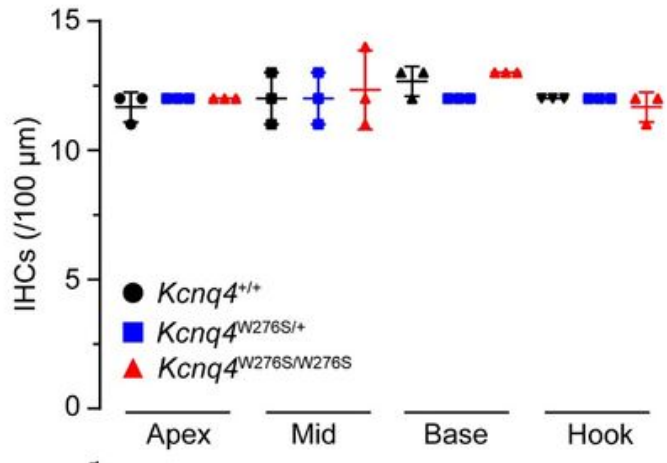

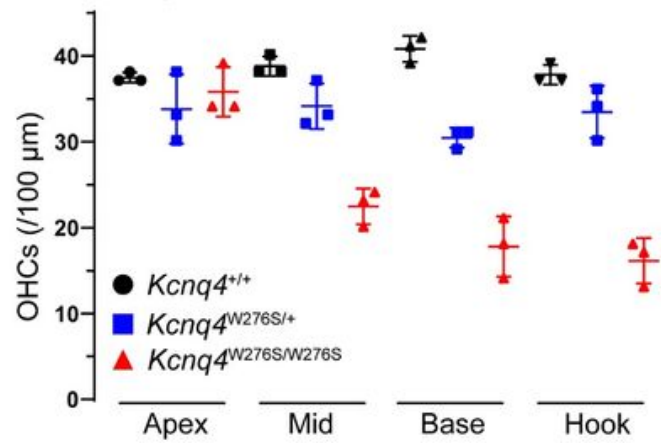

Figure 1

Mutant KCNQ4 as a common cause of adult-onset hearing loss (AHL) in humans and mice. (a) In 213 patients with $\mathrm{AHL}$, whole or targeted exome sequencing was performed. Of the 213 individuals, 33.8\% (72 patients) had a genetic diagnosis of AHL. Among them, KCNQ4 $(n=9)$ was most commonly mutated 
gene. (b) KCNQ4 expression in the basolateral surface of the outer hair cells (OHCs) in the organ of Corti of the inner ear of a 3-week-old wild-type mouse. Scale bar, $10 \mu \mathrm{m}$. (c) Sequence data for the Kcnq4 mutant mouse model used. A pathogenic missense mutation in Kcnq4 $(\mathrm{c} .830 \mathrm{G}>\mathrm{C})$ was targeted for gene editing. Prior to target mutation, an additional synonymous variant (c. $810 \mathrm{C}>\mathrm{A})$ was introduced at nucleotide position 20 because this synonymous variant was utilized for the identification of the original mutant allele after Cas 9 editing at the target mutation site; moreover, this variant prevented the Nde1 enzyme from cutting within this position, enabling the use of Nde1 restriction enzymes for genotyping heterozygote mutant mice. (d) Characteristics associated with auditory function in Kcnq4 heterozygote mutant mice. (e) Cross-sectional images of the cochlea at the apex, mid, base, and hook regions from 3week-old wild-type, heterozygote mutant, and homozygote mutant mice. Immunostaining was performed with DAPI (blue) and phalloidin (green). Scale bar, $20 \mu \mathrm{m}$. (f) Number of live inner hair cells (IHCs) and outer hair cells (OHCs) in sections indicated in (d).

a

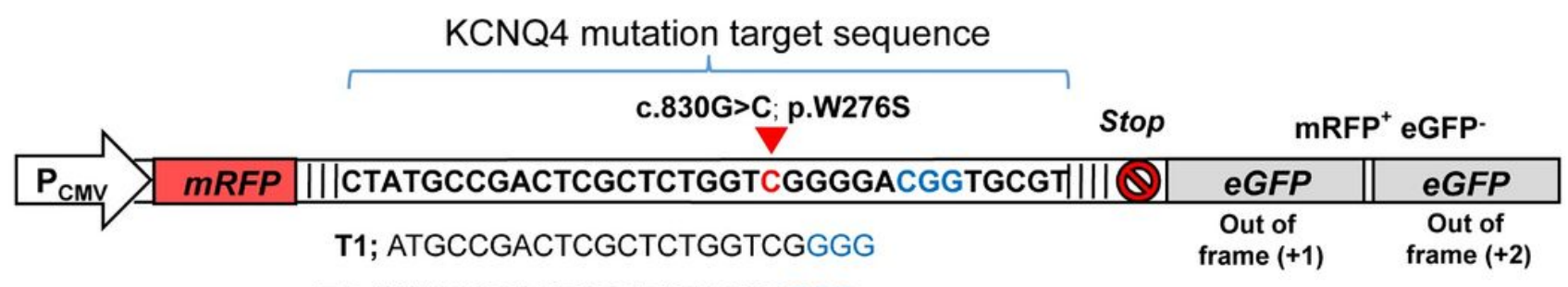

T2; TATGCCGACTCGCTCTGGTCGGG

T3; CGACTCGCTCTGGTCGGGGACGG

T4; CTATGCCGACTCGCTCTGGTCGG

b

Lenti
Reporter only

Lenti

Reporter T1

Lenti

Reporter T2

Lenti

Reporter T3

Lenti

Reporter T4
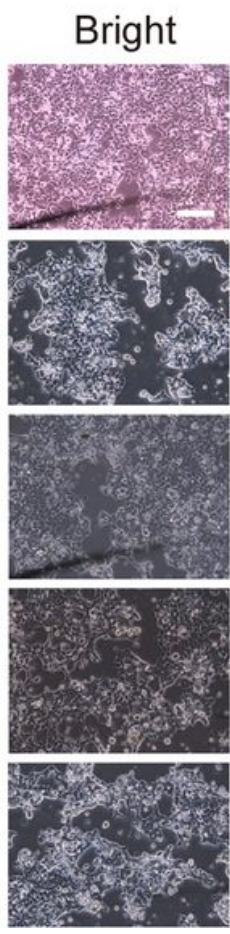
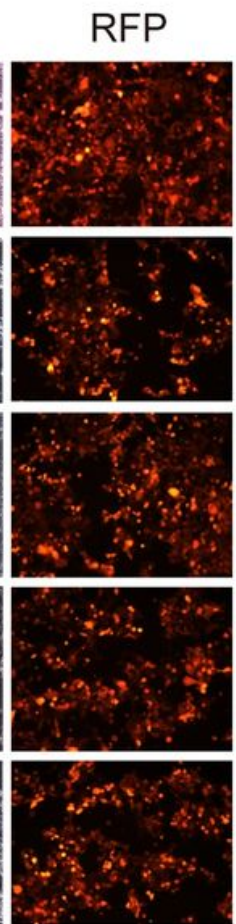

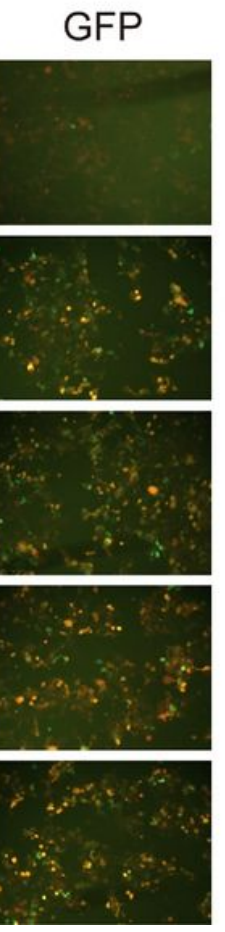

T7E1 Indel (\%) 0.034 .433 .134 .031 .1 NGS Indel (\%) 0.0522 .424 .620 .322 .7

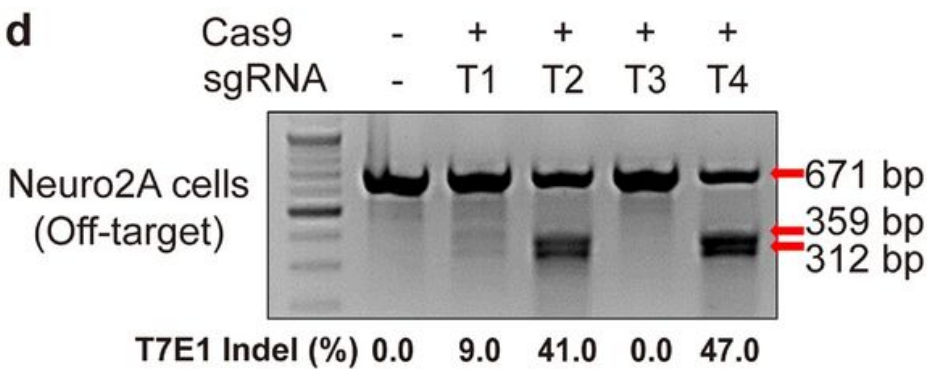


Optimization of sgRNA for in vivo gene editing. (a) Schematic representation of the reporter construct for sgRNA selection. Protospacer-adjacent motif (PAM) sequences of each sgRNA are in blue. (b) Fluorescent microscopy images of the reporter cell lines after transfection of the plasmid encoding Cas9 and each sgRNA targeting the Kcnq4 mutation target region in the reporter cells. Scale bar, $50 \mu \mathrm{m}$. (c) Target editing efficiency of sgRNA candidates determined by the T7E1 assay and deep sequencing analysis. The top band is uncut DNA at $630 \mathrm{bp}$; the two smaller cleaved bands are edited DNA (450 bp and $180 \mathrm{bp}$ ). (d) Offtarget activity screening for sgRNA candidates.

a

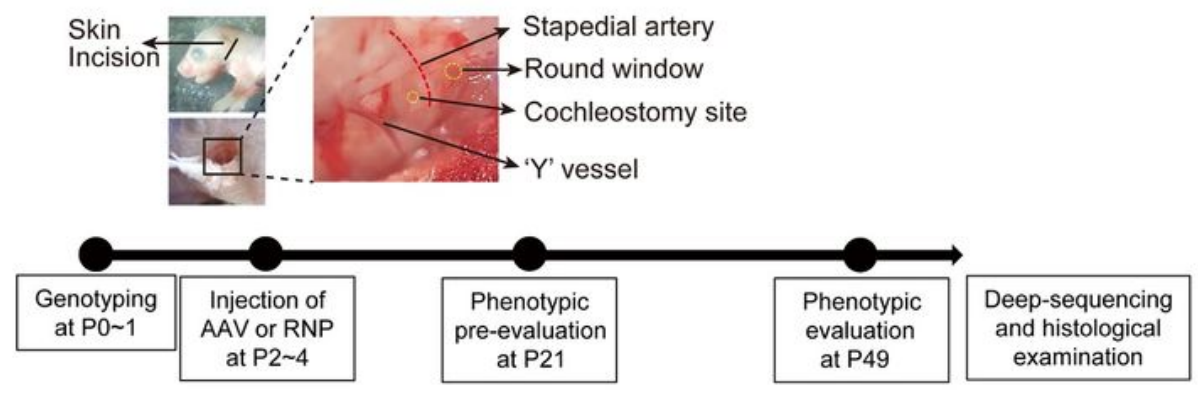

b

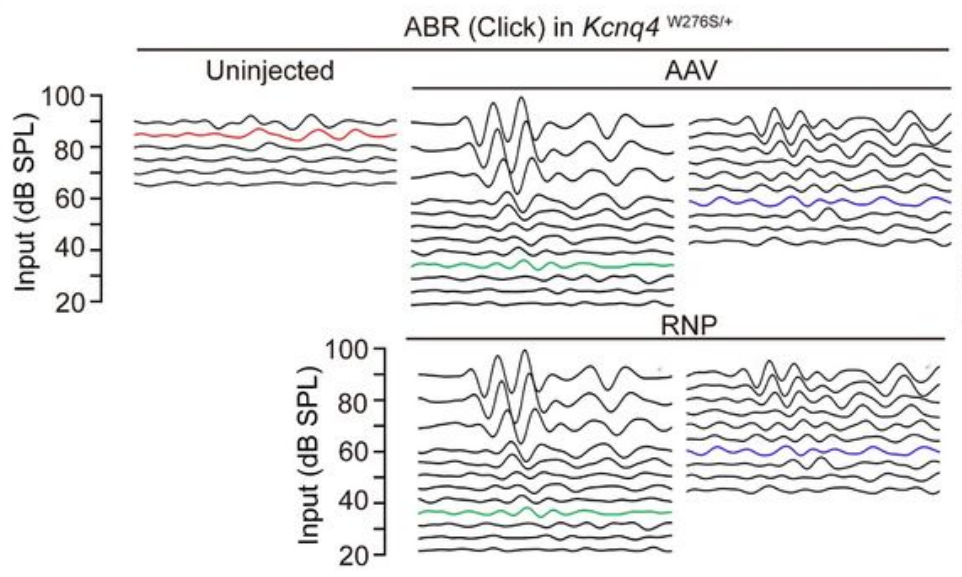
$\mathrm{ABR}$ (Click) in Kcnq4 ${ }^{+/+}$

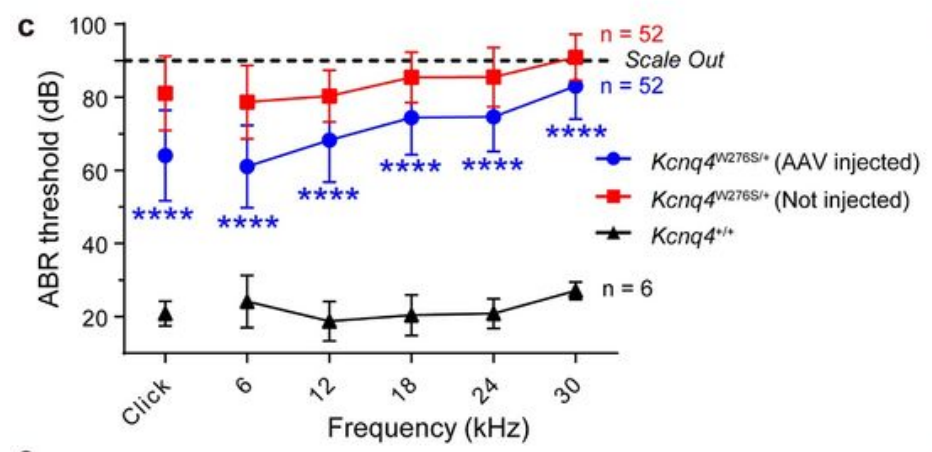

d
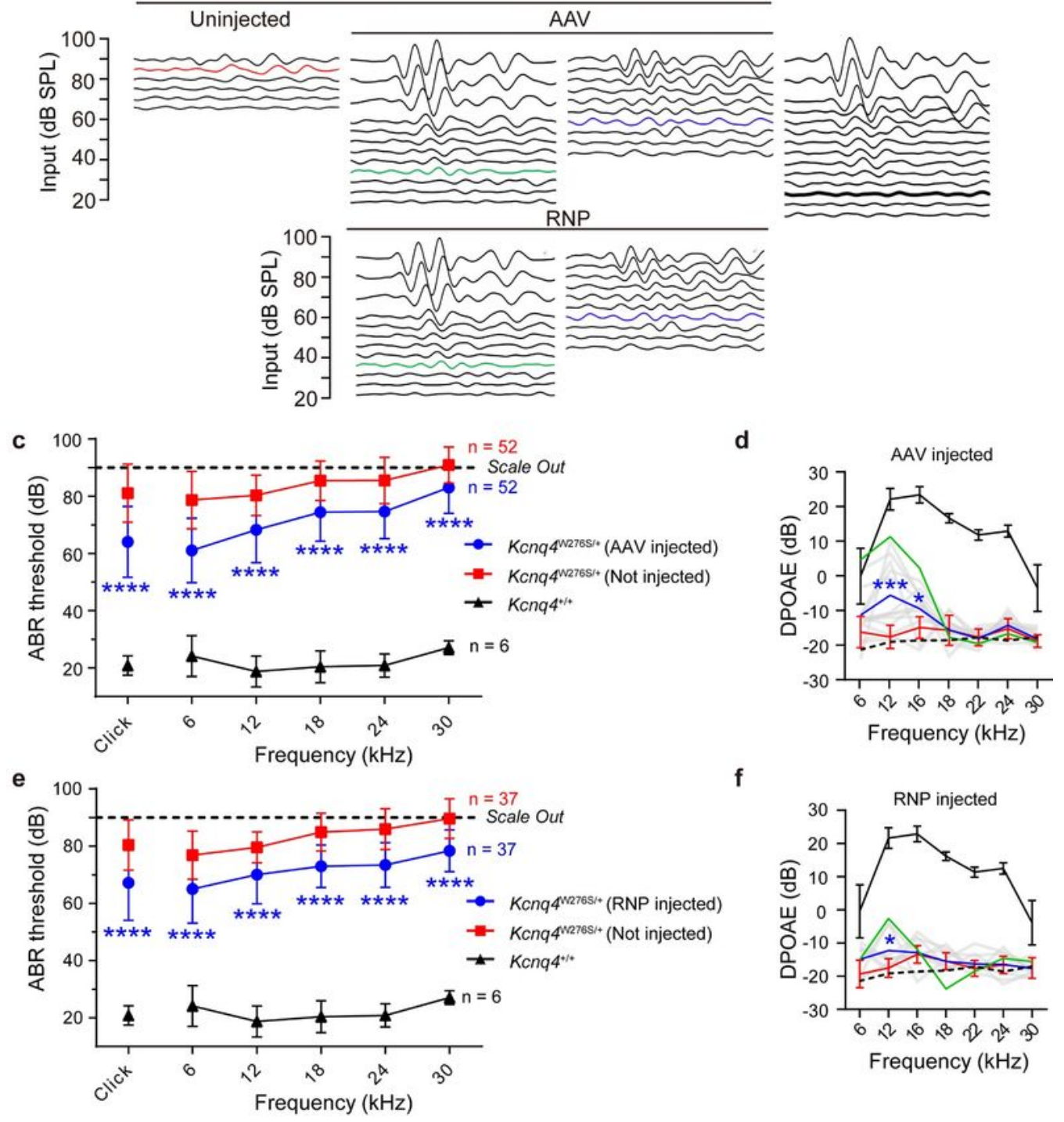


\section{Figure 3}

Phenotypic rescue of hearing loss by AAV and RNP injections in Kcnq4+/W276S mice. (a) Experimental workflow of injection procedures to examine the phenotype and determine gene editing efficacy. (b) Auditory brainstem response (ABR) waveform patterns of contralateral uninjected, AAV-injected, and RNPinjected cochlea from 7-week-old Kcnq4 mutant and wild-type mice (control). The best (green) and median (blue) recovery degrees are shown. (c) Comparative analysis of ABR thresholds across all frequencies in AAV-injected and uninjected cochlea of mutant and wild-type control mice. (d) Distortionproduct otoacoustic emission (DPOAE) thresholds in AAV-injected mutant, uninjected mutant, and wildtype mice. The best (green) and mean (blue) corrected cochleae are presented, along with the other AAVinjected cochleae (gray). (e) Comparative analysis of ABR thresholds across all frequencies in RNPinjected mutant, uninjected mutant, and wild type cochleae. (f) DPOAE thresholds of RNP-injected mutant, uninjected mutant, and wild-type mice. The best (green) and mean (blue) corrected cochleae are

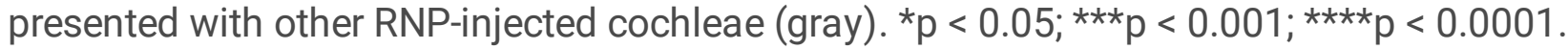


a

\begin{tabular}{|c|c|c|c|}
\hline In vivo sample & cleavage site & Count & \multirow[b]{4}{*}{$\begin{array}{l}\text { Gene editing } \\
\text { rate }\end{array}$} \\
\hline Wild type allele & СTCСTCCTATGCCGACTCGCTCTGGTGGGGGACGGTGC & 3618 & \\
\hline Mutant allele & СTCСTCATATGCCGACTCGCTCTGGTCGGGGACGGTGC & 4351 & \\
\hline $\begin{array}{l}\text { "Edited" } \\
\text { Mutant allele \#1 }\end{array}$ & СТССTCATATGCCGACTCGCTCTGGTCGGCGGACGGTGC & 11 & \\
\hline $\begin{array}{l}\text { "Edited" } \\
\text { Mutant allele \#2 } \\
\text { "Edited" } \\
\text { Mutant allele \#3 }\end{array}$ & $\begin{array}{l}\text { СTCСTCATATGCCGACTCGCTCTGGTCGGGGGACGGTGC } \\
\text { СTCСTCATATGCCGACTCGCTCTGGTCG_GGACGGTGC }\end{array}$ & 6 & \multirow[t]{2}{*}{$\begin{array}{l}(11+6+5+4) / \\
(4351+11+6+5+4) \\
=0.6 \%\end{array}$} \\
\hline $\begin{array}{l}\text { "Edited" } \\
\text { Mutant allele \#4 }\end{array}$ & СTCCTCATATGCCGACTCGCTCTGGTC-_-GGACGGTGC & 4 & \\
\hline
\end{tabular}

\begin{tabular}{|c|c|c|c|}
\hline Ex vivo sample & cleavage site & Count & \multirow[b]{3}{*}{$\begin{array}{c}\text { Gene editing } \\
\text { rate }\end{array}$} \\
\hline Wild type allele & СTCСTCCTATGCCGACTCGCTCTGGTGGGGGACGGTGC & 1179 & \\
\hline Mutant allele & CTCCTCATATGCCGACTCGCTCTGGTCGGGGACGGTGC & 1428 & \\
\hline $\begin{array}{l}\text { "Edited" } \\
\text { Mutant allele \#1 }\end{array}$ & СTCСTCATATGCCGACTCGCTCTGGTCG_GGACGGTGC & 8 & 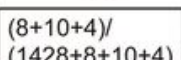 \\
\hline $\begin{array}{l}\text { "Edited" } \\
\text { Mutant allele \#2 }\end{array}$ & СТССTCATATGCCGACTCGСTCTGGTC-GGACGGTGC & 10 & $\begin{array}{l}(1428+8+10+4 \\
=1.5 \%\end{array}$ \\
\hline $\begin{array}{l}\text { "Edited" } \\
\text { Mutant allele \#3 }\end{array}$ & СTCСTCATATGCCGACTCGCTCTGGTCGGGGGACGGTGC & 4 & \\
\hline
\end{tabular}

b
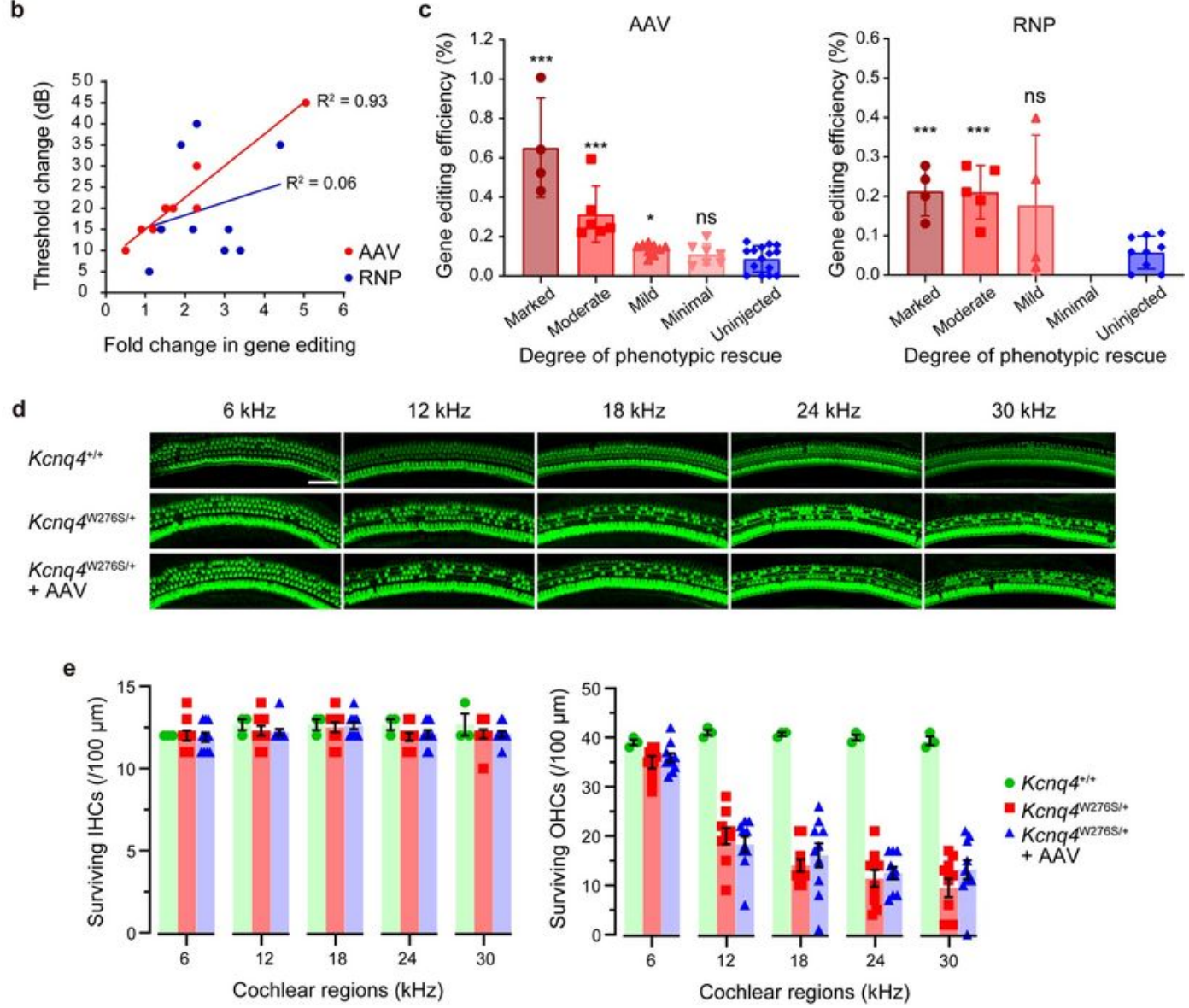

\section{Figure 4}

In vivo gene editing by AAV and RNP injection and the corresponding degree of phenotypic rescue in Kcnq4+/W276S mice. (a) Allele-specific gene editing rates obtained by deep sequencing. (b) Correlation between gene editing efficiency in terms of the fold change of correction rates and phenotypic rescue degrees evaluated by ABR threshold shift levels at click sound. Fold changes in mutant allele-specific correction rates in injected and contralateral cochleae of the same mouse showed a higher correlation 
trend $(R 2=0.93)$ with ABR threshold shift in AAV than in RNP $(R 2=0.06)$. (c) Gene editing efficiency according to phenotypic rescue degrees evaluated by ABR threshold shift levels across all frequencies. Phenotypic rescue degrees were classified into four subgroups based on average $\mathrm{dB}$ differences between injected and contralateral cochleae at five frequencies $(6,12,18,24$, and $30 \mathrm{kHz})$ : marked: $\geq 15 \mathrm{~dB}$; moderate: $10-14 \mathrm{~dB}$; mild: 5-9 dB; and minimal: $\leq 5 \mathrm{~dB}$ ). ${ }^{*} \mathrm{p}<0.05$; ${ }^{\star \star} \mathrm{p}<0.001$; ns, not significant. (d) Hair cell survival patterns in the cochleae of wild-type, uninjected mutant, and injected mutant across five frequencies after immunostaining with phalloidin (green). Scale bar, $50 \mu \mathrm{m}$. (e) Number of live inner and outer hair cells in the cochleae of wild-type, uninjected mutant, and injected mutant mice across five frequencies $(p>0.05)$.

a
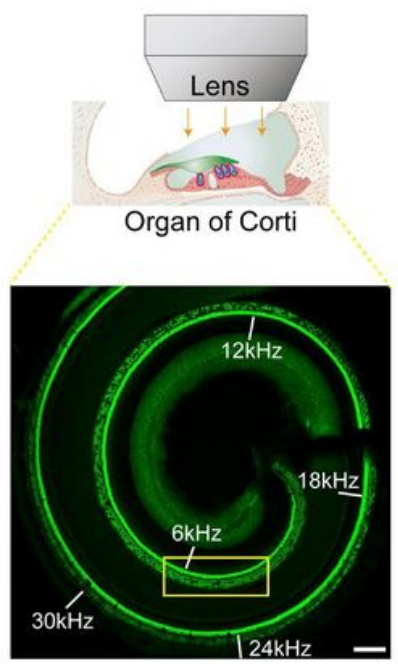

d

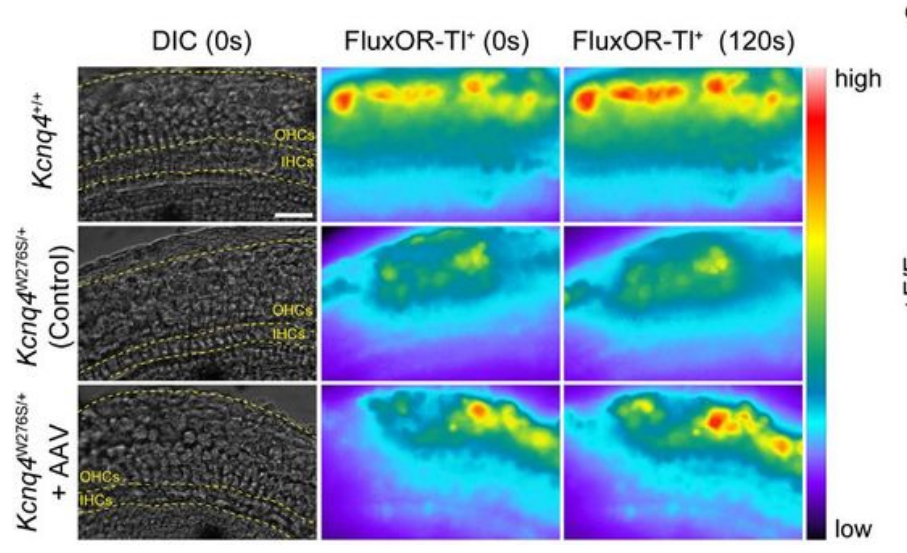

b
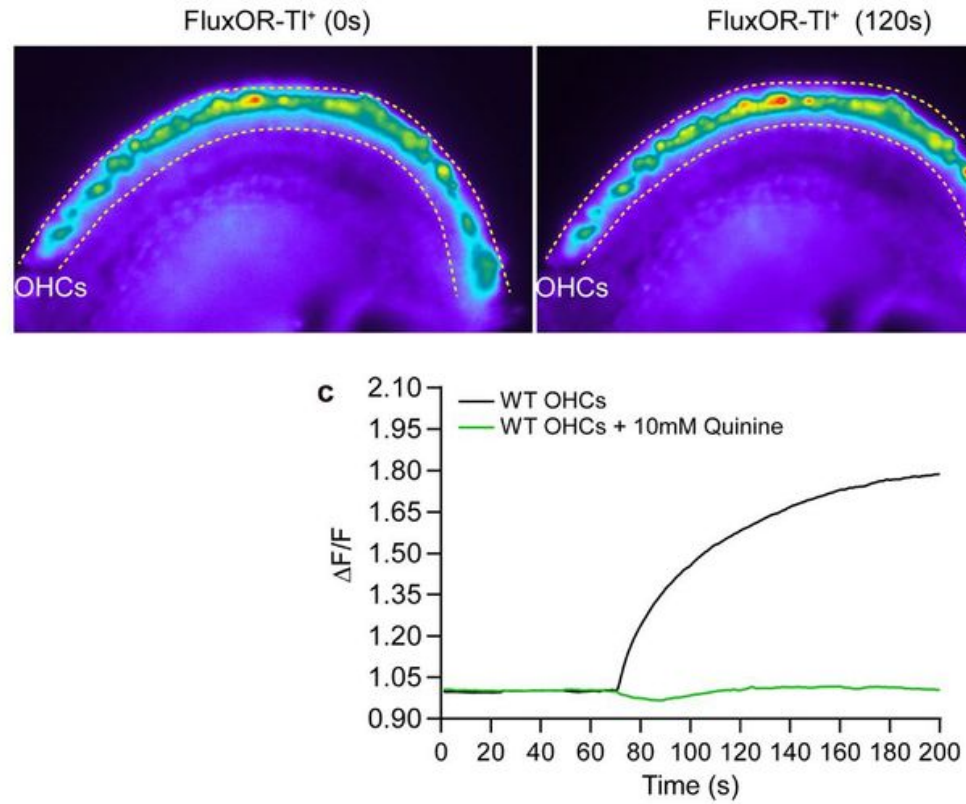

e

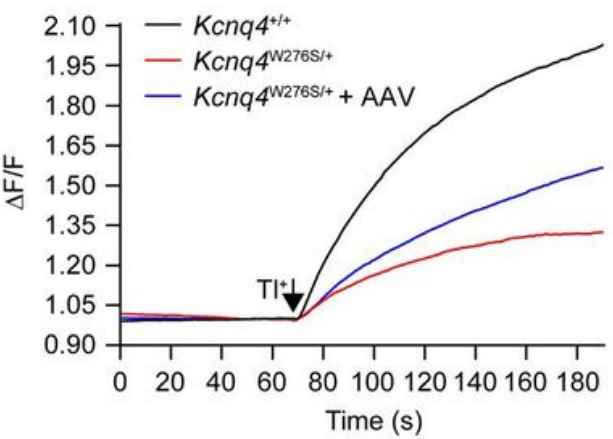

f

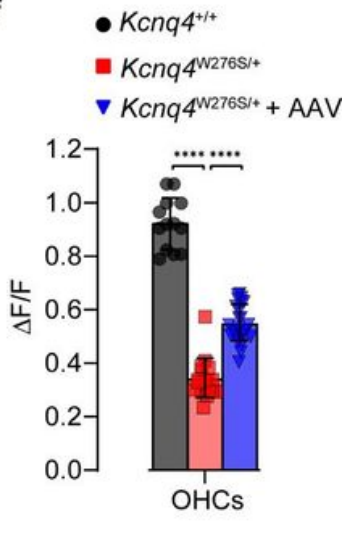

\section{Figure 5}

Mechanisms responsible for phenotypic rescue in Kcnq4+/W276S mice in response to gene editing. (a) Cochlea stained with phalloidin (green) of AAV-injected mutant mice euthanized at 7 weeks. Tonotopical regions are marked with the corresponding frequencies (white); TI+ flux assay was performed at the 6 $\mathrm{kHz}$ region under a microscope. Scale bar, $100 \mu \mathrm{m}$. (b) Microscopy images of the organ of Corti (apex) of 7-week-old wild type mouse before the addition of TI+ and after 120 seconds. (c) Changes in fluorescence 
intensity values in the 6-kHz region of the cochlea of 7-week-old wild-type mice after the addition of the stimulus buffer, including $\mathrm{Tl}+$, during the thallium flux assay. In the negative control, $10 \mathrm{mM}$ of quinine, which inhibits the mechano-electrical transducer channel, inhibited the influx of Tl+ into the hair cells of wild-type cochlea. (d) Changes in fluorescence intensity before and $120 \mathrm{~s}$ after the addition of thallium in the contralateral cochleae of uninjected and AAV-injected mice. The yellow dotted line shows the boundary between the inner (IHCs) and outer hair cells (OHCs). Scale bar, $50 \mu \mathrm{m}$. (e) Sequential changes in $\mathrm{F} / \mathrm{F}$ values after $\mathrm{TI}+$ stimulus in the IHCs and OHCs of the cochleae of wild-type, AAV-injected mutant, and uninjected mutant mice. (f) Comparative analysis of the change in the slope of the fluorescence density of the IHCs and OHCs of wild-type, injected mutant, and uninjected mutant mice. ${ }^{\star \star \star \star} p<0.001$. 


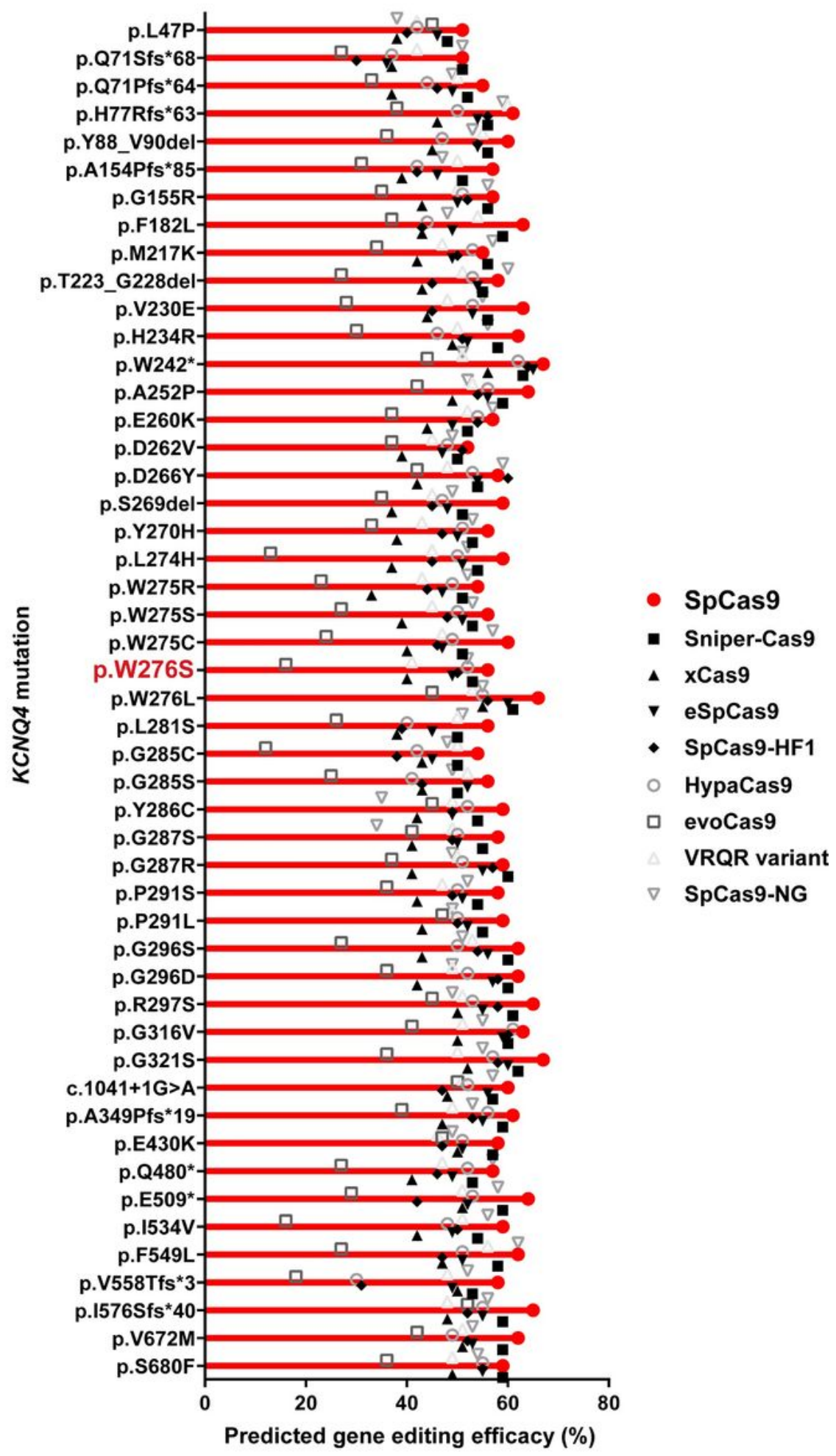

Figure 6

In vivo gene editing scores for mutations in KCNQ4. In total, 49 mutations linked to DFNA2 were selected to evaluate the in vivo gene editing scores. Missense and frameshift mutations, as well as splicing variants, were included. The DeepSpCas9 prediction program was utilized to test the predicted efficiency of in vivo gene editing based on combinations of candidate sgRNAs for KCNQ4 mutant sequences and Cas9 variant forms. 


\section{Supplementary Files}

This is a list of supplementary files associated with this preprint. Click to download.

- SupplementaryMovie1.avi

- Supplementaryinformation.pdf

- SupplementaryTables14.xlsx 